\title{
A Ritz Approximation to the Deformation of Anisotropic Slender Structures with Finite-Size Cross Sections
}

\author{
Rafael Palacios ${ }^{1}$ and Carlos E.S. Cesnik ${ }^{2}$ \\ University of Michigan, Ann Arbor, Michigan, 48109-2140, USA
}

\begin{abstract}
A one-dimensional theory of slender structures with heterogeneous anisotropic materials is presented. It expands Cosserat's description of beam kinematics by allowing deformation of the beam cross sections. For that purpose, a Ritz approximation is introduced on the cross-sectional warping field, which defines additional elastic degrees of freedom (finitesection modes) in the 1-D model. This results in an extended set of beam dynamic equations that includes direct measures of both the large global displacement and rotations of a certain reference line, and the small local deformations of the cross sections. Two situations of interest are then studied in which this approach provides a simpler alternative to nonlinear shell models: First, we look at the detailed structural response of thin-walled composite beams with distributed loads. In particular, the case of a composite construction with embedded piezoelectric actuators is considered. Second, this methodology is applied to study the low-frequency response characterization of a thin-walled composite beam. Numerical results are presented in both cases, in which a reduced set of finite-section modes allows a full characterization of the actual 3-D structure within a strictly 1-D framework solution.
\end{abstract}

\section{Nomenclature}

$\begin{array}{ll}\overline{\delta \Phi} & =\text { column matrix of local virtual rotations of reference line } \\ \overline{\delta \varphi} & =\text { column matrix of virtual rotations of reference coordinate system } \\ \boldsymbol{\Gamma} & =\text { strain tensor in the } 3 \text {-D solid domain } \\ \boldsymbol{\gamma} & =\text { vector of force strain measures at reference line } \\ \boldsymbol{\kappa} & =\text { vector of moment strain measures at reference line } \\ \boldsymbol{\mu} & =\text { vector of distributed applied force per unit volume } \\ \rho & =\text { mass density } \\ \boldsymbol{\Omega} & =\text { inertial angular velocity vector at the deformed reference line } \\ \boldsymbol{\omega} & =\text { inertial angular velocity vector of the reference coordinate system }(a) \\ \Psi & =\text { matrix of approximating functions for finite-section modes } \\ \overline{\delta \mathcal{A}} & =\text { applied action at time and spatial boundaries } \\ A & =\text { area of the cross section }\end{array}$

\footnotetext{
${ }^{1}$ Postdoctoral Research Fellow (rpalacio@umich.edu), Department of Aerospace Engineering, AIAA Member

${ }^{2}$ Associate Professor (cesnik@umich.edu), Department of Aerospace Engineering, Associate Fellow, AIAA 


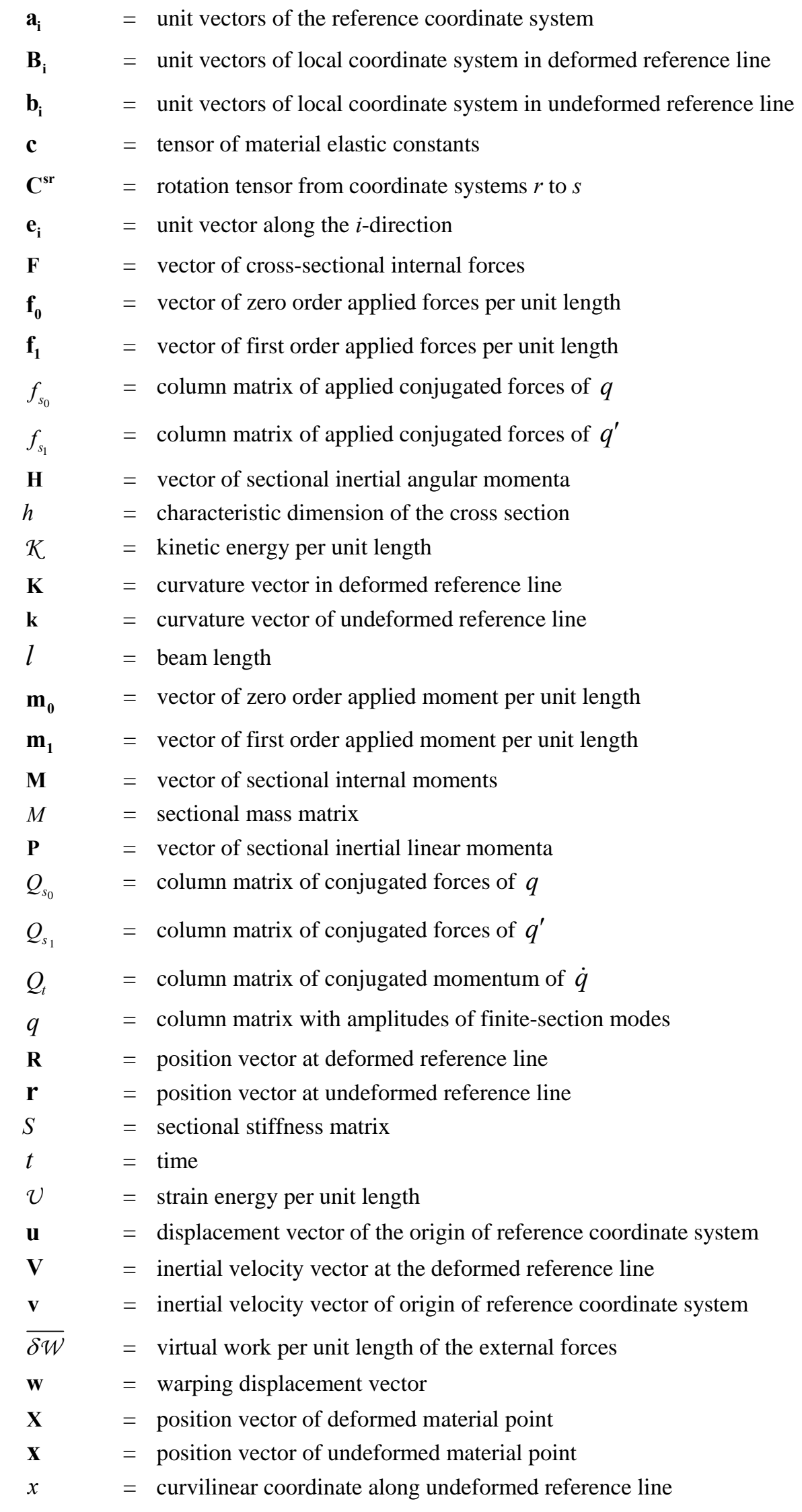




$\begin{array}{ll}x_{\alpha} & =\text { cross-sectional coordinates in the reference configuration } \\ \delta & =\text { variational operator } \\ (\bullet) & =\text { cross-product operator } \\ (\bullet) & =\text { differentiation with respect to } t \\ (\bullet)^{\prime} & =\text { differentiation with respect to } x\end{array}$

\section{Introduction}

$\mathrm{W}$

hile beam models allow the easy modeling of structural elements with a dominant spatial dimension, their application is typically limited by additional kinematic constraints to the deformation. One typical assumption is that the deformation of the beam reference line defines the displacement field in the actual 3-D slender solid, by assuming, for instance, rigid cross sections (Cosserat's model). The resulting models are valid while the area of the cross-section remains close to zero, but it is not that obvious to establish their limits when the crosssectional size becomes important. The aim of this paper is then to establish a theory of beams with finite-size cross sections that satisfies the following requirements: 1) it uses a 1-D representation of the structure; 2) it allows for deformations of the cross section not necessarily related to the deformation of the reference line; 3) it does not impose additional modeling restrictions (it accounts for cross sections of arbitrary shape, material distribution, and curved reference lines). Furthermore, such beam description should account for arbitrarily large deformations of the reference line.

Basic concepts of beam theory were first developed for the modeling of solid homogeneous isotropic structures. There we find the classical linear beam theories used in strength of materials, i.e., Euler-Bernoulli or Timoshenko theories. They are displacement formulations: an ad hoc approximation of the cross-sectional displacement field yields the strain energy and, using energy principles, some beam stiffness relations can be defined, as well as the subsequent equations of motion. Those classical assumptions in the displacement field, however, no longer represent the behavior of anisotropic slender structures. The concept of warping, which was first developed for the SaintVenant theory of torsion, is used then to quantify this mismatch, and based on it, a number of composite beam theories have been formulated. With an interest in helicopter blade design, Volovoi et al. ${ }^{1}$ and Jung et al. ${ }^{2}$ have recently reviewed the available modeling methods for anisotropic beams.

Asymptotic expansions provide the mathematical tool to constructing 1-D structural models from the 3-D equations of elasticity, without $a d$ hoc assumptions in the displacement field. The slenderness of the structure provides a small parameter ( $h / L$, with $h$ being the cross-sectional typical dimension, and $L$ the characteristic wavelength of the deformation) to build an asymptotic approximation to the solution of the 3-D elastic problem. In this case, there are no previous assumptions about the displacement field, which is approximated as part of the asymptotic solution. The problem can be posed initially either in its weak (Berdichevsky ${ }^{3}$, Cesnik and Hodges ${ }^{4}$ ) or strong form (Buannic and Cartraud ${ }^{6}$, Fan and Widera ${ }^{7}$, Parker $^{8}$ ). Its solution, for slender solids with cross sections of arbitrary shape, can only be obtained numerically. The problem in isotropic beams was defined by Saint-Venant as a 2-D problem in the cross section under the assumption of negligible normal stresses (as described by Love ${ }^{9}$ ), and different numerical solutions for general cross sections have been presented (e.g., finite elements in Gruttman and Wagner ${ }^{10}$ and the boundary-element method in Friedman and Kosmatka ${ }^{11}$ ). For general anisotropic structures Kosmatka $^{12}$, Giavotto et al. ${ }^{13}$, and Cesnik and Hodges ${ }^{4}$, among others, have presented reduction models based on local 3-D equilibrium equations per unit length of the beam. In Ref. 12, the warping field is obtained through minimization of the energy, but the cross-sectional and longitudinal problems are coupled in the solution, which complicates the actual implementation of the method. This is avoided in Ref. 13 through the identification of the beam motions as the kernel of the equations in the interior of the solid, what implies a variational definition of the 1D elastic properties for prismatic beams without assumptions on the deformation field. Finally, Ref. 4 applied to anisotropic beams the variational-asymptotic beam cross-sectional (VABS) analysis derived by Berdichevsky ${ }^{3}$. The big advantage of the variational description in VABS analyses is that it provides a robust framework for expansion of the formulation. Thus the original generalized Euler-Bernoulli description of Ref. 4, which is based on four classical elastic degrees of freedom (extension, twist and bending in two directions), was later expanded in Ref. 14 to account for any number of additional arbitrary non-classical 1-D elastic (and electric) degrees of freedom. These non-classical deformations include situations such as the camber bending deformation of thin strips (Palacios and Cesnik $^{15}$ ) or the generalization of the Vlasov correction for open-cell composite beams of Volovoi and Hodges ${ }^{16}$. 
This results in a theory of beams with arbitrary deformable cross-sections, which will be fully developed in this work.

The formulation in this work will reduce the description of the solid motion to the evolution of certain magnitudes along a reference line. This reference line will be allowed to move in space and have arbitrarily large deformations. Geometrically nonlinear analysis of beams usually follows Cosserat's model: the beam is represented by a deformable curve in space with cross sections that move rigidly with it. The elastic properties are assumed to be known and the deformation of the curve is analyzed using the tools of differential geometry, including its definition through a set of intrinsic kinematical quantities. Kirchhoff and Clebsch (Love ${ }^{9}$, article 254) already determined the geometrically-exact equations of equilibrium in flexible beams that relate the longitudinal variation of resultant forces with the curvatures of the reference line. The effect of shear deformation was later considered by Reissner ${ }^{17}$, who also presented a definition of virtual rotations to obtain the intrinsic equations of static equilibrium from variational principles. A geometrically-exact model for beam dynamics was later introduced by Simo ${ }^{18}$. Those are intrinsic formulations, but are explicitly solved in displacements/rotations. In particular, the description of the

kinematics used rotation tensors. An important simplification was introduced later by Danielson and Hodges ${ }^{19-20}$, who developed a decomposition of the rotations in their global and local components. Based on this approach, Hodges $^{21}$ derived a mixed form of the equations for beam dynamics, which is solved in a very simple numerical scheme in Hodges et al. $^{22}$. Alternatively, strain-based solutions to the geometrically-exact problem have been proposed recently by Cesnik and Brown ${ }^{23}$ and Hodges ${ }^{25}$.

The intrinsic beam model of Hodges ${ }^{21}$ is used here to describe the geometrically-nonlinear deformations of the reference line. It is expanded by the non-classical (finite-section) modes introduced in Ref. 14 to provide a more accurate description of the actual deformation in the 3-D solid. Finally, the theory is set up in the context of linear piezoelasticity to account for embedded actuation in the composite construction.

\section{Theoretical Development}

Starting from the elastodynamic equations of the 3-D elastic solid, the slenderness of the structure is used to define a small parameter in the equations. Two scales are then identified in the analysis and the problem is split into a long-scale problem along the longitudinal dimension, and a small-scale problem at each cross section. A previous work by the authors ${ }^{14}$ has presented the general solution to the cross-sectional problem and this work will focus on the details on the development of the one-dimensional dynamic equations along the reference line. For completeness, some key results on the reduction process from the 3-D solid to the reference line are also included here.

\section{A. Kinematics}

Consider the two configuration states in the deformation of a slender solid of slowly-varying cross section shown in Figure 1. They correspond to the undeformed (reference) and deformed (current) configurations. The deformation is described using a Lagrangian description, with lower- and upper-case symbols used for magnitudes at the undeformed and deformed state, respectively. The description is done from the viewpoint of an observer on a moving reference frame $(a)$. The motion of this frame is given with respect to an inertial frame (a certain global frame, $o$ ) by the translational velocity of its origin, $\mathbf{v}$, and its rotational velocity, $\boldsymbol{\omega}$, which, in general, are unknown.

A (curved) reference line, $r$, is defined along the longitudinal dimension of the undeformed configuration. Let $\mathbf{r}$ and $\mathbf{x}$ be the position vectors along the reference line and in the undeformed solid, respectively. Curvilinear coordinates can be defined such as $x=x^{1}$ is the coordinate along $r$ and $x_{\alpha}=x^{\alpha}$ are the orthogonal coordinates in its normal planes. The associated (orthogonal) covariant base vectors are defined as

$$
\mathbf{g}_{\mathbf{i}}\left(x, x_{2}, x_{3}\right)=\frac{\partial \mathbf{x}}{\partial x^{i}} .
$$

When restrained to the points along $r$, these base vectors define the undeformed reference frame, $b$, as $\mathbf{b}_{\mathbf{i}}(x)=\mathbf{g}_{\mathbf{i}}(x, 0,0)$. The position vector $\mathbf{x}$ in the undeformed solid can be written as

$$
\mathbf{x}\left(x, x_{2}, x_{3}\right)=\mathbf{r}(x)+x_{\alpha} \mathbf{b}_{\boldsymbol{\alpha}}(x) .
$$




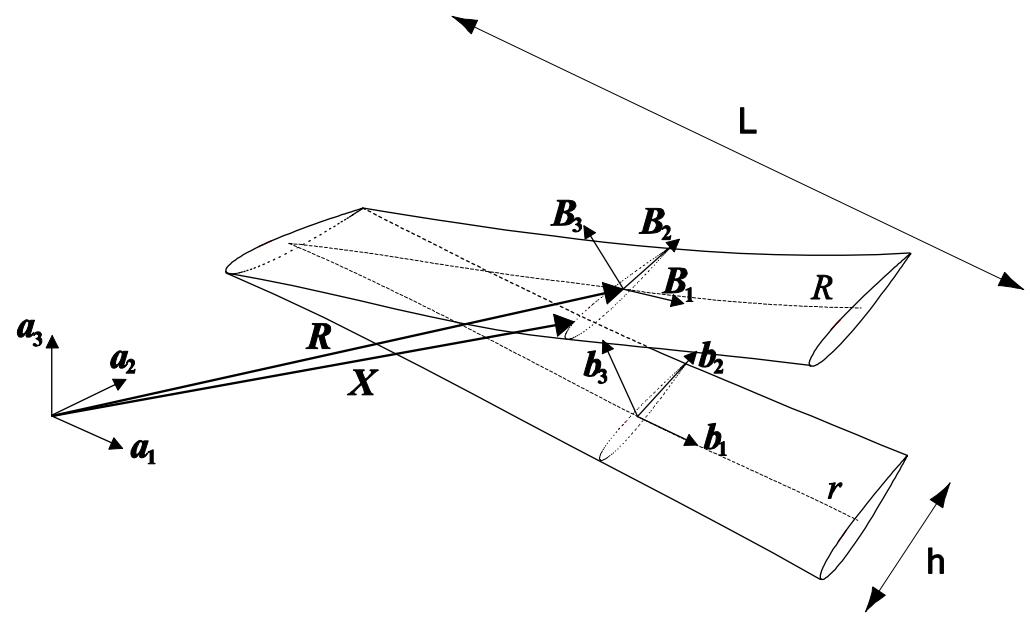

Figure 1. Coordinate frames in the deformation of a slender solid.

Let $\mathbf{X}$ be the position vector in the current configuration of a material point initially at $\mathbf{x}$. We define now the deformed reference line, $R$, by the averaged value of $\mathbf{X}$ on the cross sections of $r$

$$
\mathbf{R}(x)=\frac{1}{A(x)} \int_{A(x)} \mathbf{X} d A,
$$

where $A(x)$ is the cross-sectional area at $x$. The covariant base vectors in the deformed domain are

$$
\mathbf{G}_{\mathbf{i}}\left(x, x_{2}, x_{3}\right)=\frac{\partial \mathbf{X}}{\partial x^{i}},
$$

which do not define, in general, an orthogonal basis. Therefore, to simplify the description we introduce an "intrinsic" orthogonal deformed reference frame, $B$, which is not necessarily equal to $\mathbf{G}_{\mathbf{i}}(x, 0,0)$. Defining $\mathbf{Z}=\mathbf{X}-\mathbf{R}$ as the position vector in the deformed cross section, the deformed frame is defined by enforcing that the average rotation around the three vectors $\mathbf{B}_{\mathbf{i}}$ is zero, that is,

$$
\begin{gathered}
\int_{A(x)}\left(x_{3} \mathrm{Z}_{2}-x_{2} \mathrm{Z}_{3}\right) d A=0, \\
\int_{A(x)} x_{3} \mathrm{Z}_{1} d A=0, \\
\int_{A(x)} x_{2} \mathrm{Z}_{1} d A=0 .
\end{gathered}
$$

where $\mathbf{Z}=Z_{i} \mathbf{B}_{\mathbf{i}}$. The rotation matrices between the global frame $(a)$ and the undeformed $(b)$ and deformed $(B)$ frames will be denoted as $C^{b a}(x, t)$ and $C^{B a}(x, t)$, respectively. Their spatial derivative along the reference line defines the curvature vectors of the undeformed $\left(\mathbf{k}=k_{b, i} \mathbf{b}_{\mathbf{i}}\right)$ and deformed $\left(\mathbf{K}=K_{B, i} \mathbf{B}_{\mathbf{i}}\right)$ reference lines, respectively, as

$$
\tilde{k}_{b}=-\left(C^{b a}\right)^{\prime} C^{a b}, \quad \tilde{K}_{B}=-\left(C^{B a}\right)^{\prime} C^{a B} .
$$

The position vector in the current configuration of the material point initially defined by (2) is then written, without loss of generality, as ${ }^{14}$ 


$$
\mathbf{X}\left(x, x_{2}, x_{3}\right)=\mathbf{R}(x)+x_{\alpha} \mathbf{B}_{\boldsymbol{\alpha}}(x)+q_{n}(x) \Psi_{n, i}\left(x_{2}, x_{3}\right) \mathbf{B}_{\mathbf{i}}(x)+w_{i}\left(x, x_{2}, x_{3}\right) \mathbf{B}_{\mathbf{i}}(x),
$$

where $\Psi_{n, i}$, with $n=1, \ldots, N$, is a set of approximating functions to the sectional deformation field to capture "nonclassical" deformations (which are referred to in what follows as finite-section modes), $q_{\boldsymbol{n}}$ are the amplitudes of those finite-section modes, and $\mathbf{w}=w_{i} \mathbf{B}_{\mathbf{i}}$ is the residual warping displacement vector. Note that, if the finite-section modes are zero and the warping is the (prescribed) Saint-Venant torsion warping, Eqs. (5) and (7) correspond to the kinematic assumptions for the Timoshenko model of an isotropic beam with extension, bending, shear and twist degrees of freedom. In the general case, for other values of these variables, several orthogonality constraints need to be imposed on them to give a unique description of the deformation. They are chosen as ${ }^{14}$

$$
\begin{gathered}
\int_{A(x)} \bar{\Psi}^{T} w d A=0, \\
\int_{A(x)} \Psi^{T} \Psi_{0} d A=0,
\end{gathered}
$$

where

$$
\Psi_{0}=\left[\begin{array}{cccccc}
1 & 0 & 0 & -x_{2} & x_{3} & 0 \\
0 & 1 & 0 & 0 & 0 & -x_{3} \\
0 & 0 & 1 & 0 & 0 & x_{2}
\end{array}\right], \quad \bar{\Psi}=\left[\begin{array}{lll}
\Psi_{0} & \mid \Psi
\end{array}\right], \text { and } \quad w=\left\{\begin{array}{l}
w_{1} \\
w_{2} \\
w_{3}
\end{array}\right\}
$$

\section{B. Equations of motion}

The equations of motion of the linear elastic domain are given by Hamilton's principle applied in a time interval $\left[t_{1}, t_{2}\right]$ and in the spatial domain $\Omega$, as

$$
\int_{t_{1}}^{t_{2}}\left\{\int_{\Omega}[\delta(\kappa-u)+\overline{\delta w}] d \mathbf{x}\right\} d t=\overline{\delta \mathcal{A}}
$$

where $\overline{\delta w}$ is the virtual work per unit volume of the external forces, $\overline{\delta \mathcal{A}}$ includes any virtual action on the boundaries of the domain $\Omega$ and at the ends of the time interval, and $k$ and $u$ are the kinetic and strain energy densities, respectively. Overbars on virtual magnitudes indicate that they do not correspond to the variation of a function. Both energy densities are given, respectively, by

$$
\begin{aligned}
& k=\frac{1}{2} \rho \dot{\mathbf{X}} \cdot \dot{\mathbf{X}}, \\
& u=\frac{1}{2} \boldsymbol{\Gamma} \cdot \mathbf{c} \cdot \boldsymbol{\Gamma},
\end{aligned}
$$

where $\rho$ is the material density and $\boldsymbol{c}$ is the fourth-order tensor of elastic material constants (compliances). $\boldsymbol{\Gamma}$ is the local Jaumann-Biot-Cauchy strain tensor, given in a mixed-bases projection as in Danielson and Hodges ${ }^{19}$,

$$
\Gamma_{i j}=\frac{1}{2}\left(A_{i j}+A_{j i}\right)-\delta_{i j} \text {, with } A_{i j}=\mathbf{B}_{\mathbf{i}} \cdot \frac{\partial \mathbf{X}}{\partial \mathbf{x}} \cdot \mathbf{b}_{\mathbf{j}} .
$$

Spatial differentiation of Eq. (7) gives the local strain tensor in the solid as

$$
\boldsymbol{\Gamma}=\boldsymbol{\Gamma}_{\gamma} \gamma(x, t)+\boldsymbol{\Gamma}_{\mathbf{\kappa}} \kappa(x, t)+\boldsymbol{\Gamma}_{\mathbf{q}_{\mathbf{n}}} q_{n}(x, t)+\boldsymbol{\Gamma}_{\mathbf{q}_{\mathbf{n}}^{\prime}} q_{n}^{\prime}(x, t)+\boldsymbol{\Gamma}_{\mathbf{w}} w+\boldsymbol{\Gamma}_{\mathbf{1}} w^{\prime},
$$


where $\boldsymbol{\Gamma}_{(\bullet)}$ are a set of linear operators defined by Cesnik and Hodges ${ }^{4}$ (with $\boldsymbol{\Gamma}_{\mathrm{q}_{\mathrm{n}}}=\boldsymbol{\Gamma}_{\mathrm{w}} \cdot \boldsymbol{\Psi}_{\mathrm{n}}$, and $\boldsymbol{\Gamma}_{\mathrm{q}_{\mathrm{n}}^{\prime}}=\boldsymbol{\Gamma}_{1} \cdot \boldsymbol{\Psi}_{\mathrm{n}}$ ) and $\gamma$ and $\kappa$ are the column matrix form of the force and moment generalized strains, respectively, defined as ${ }^{5}$

$$
\gamma=\left\{\begin{array}{l}
\gamma_{11} \\
2 \gamma_{12} \\
2 \gamma_{13}
\end{array}\right\}=C^{B a} R_{a}^{\prime}-C^{b a} r_{a}^{\prime}, \quad \kappa=\left\{\begin{array}{l}
\kappa_{1} \\
\kappa_{2} \\
\kappa_{3}
\end{array}\right\}=K_{B}-k_{b} .
$$

Analogously, time differentiation of Eq. (7) defines the local velocities in the solid. The local inertial velocity vector $\dot{\mathbf{X}}$ is then obtained as

$$
\dot{\mathbf{X}}=\mathbf{V}+\boldsymbol{\Omega} \times\left(x_{\alpha} \mathbf{B}_{\alpha}+\boldsymbol{\Psi}_{\mathrm{n}} q_{n}+\mathbf{w}\right)+\left(\boldsymbol{\Psi}_{\mathbf{n}} \dot{q}_{n}+\dot{\mathbf{w}}\right),
$$

where the components of the translational, $\mathbf{V}=V_{B, i} \mathbf{B}_{\mathbf{i}}$, and rotational, $\mathbf{\Omega}=\Omega_{B, i} \mathbf{B}_{\mathbf{i}}$, velocity vectors (defined with respect to an inertial reference frame) are given by the following kinematical relations,

$$
\begin{gathered}
V_{B}=C^{B a}\left(\dot{R}_{a}+\tilde{\omega}_{a} R_{a}+v_{a}\right), \\
\tilde{\Omega}_{B}=-\dot{C}^{B a} C^{a B}+C^{B a} \tilde{\omega}_{a} C^{a B} .
\end{gathered}
$$

Sub-indexes in the vectors refer to the reference system in which their components are known. Substituting the strain and velocity fields of Eqs. (15) and (17), respectively, into the strain and kinetic energy densities, the variational problem of Eq. (11) defines, without any approximation, the dynamics of a slender solid in two set of variables:

1) The averaged variables along the reference line $\left\{\mathbf{R}, \mathbf{C}^{\mathrm{Ba}}, q_{n} ; \gamma, \mathbf{\kappa}, q_{n}^{\prime} ; \mathbf{V}, \boldsymbol{\Omega}, \dot{q}_{n}\right\}$, which are only spatial functions of the longitudinal coordinate, $x$, and are thus the long-scale variables.

2) The residual local warping field and its derivatives $\left\{\mathbf{w}, \mathbf{w}^{\prime}, \dot{\mathbf{w}}\right\}$, which will be referred to as small-scale variables.

The condition of slenderness, $h / L \ll 1$, ensures that the energy contributions of these small-scale variables in the 3-D dynamic equations (11) will be small compared with those of the long-scale variables, and so it effectively defines a multiscale problem in the slender solid. As a result, one can find an asymptotic approximation to the variational 3-D problem by successively solving the problem at the different scales. This methodology is known as the variational-asymptotic method ${ }^{3}$. A relatively recent book by $\mathrm{Le}^{26}$ provides a good introduction to the topic.

\section{Cross-Sectional Analysis}

The solution to the cross-sectional (small-scale) problem in the present scope was presented in Ref. 14. It is assumed that the contributions of $\dot{\mathbf{w}}$ and $\boldsymbol{\Omega} \times\left(\boldsymbol{\Psi}_{\mathbf{n}} q_{n}+\mathbf{w}\right)$ to the kinetic energy are negligible, what effectively reduces the small-scale problem to the minimization of the strain energy density at each cross section as function of the (small) local warping displacements. The small-scale problem becomes then a constrained minimization problem, defined as

$$
\delta \int_{A(x)}\left[u\left(\hat{\gamma}, \hat{\kappa}, \hat{q}_{n}, \hat{q}_{n}^{\prime} ; w, w^{\prime}\right)+\lambda \bar{\Psi} \cdot w\right] d A=0
$$

where the symbol $\hat{\bullet}$ was introduced to denote the prescribed variables in the cross-sectional problem and $\lambda$ are the Lagrange multipliers associated with the orthogonality constraints imposed by Eq. (8). A detailed analysis of the different contributions in Eq. (19) leads to the identification of different orders in the equation (i.e., $h / L$, $(h / L)^{2}$, etc.). A solution is then obtained by approximating the warping with an asymptotic expansion in the small parameter $h / L$, as

$$
w=w_{0}+\frac{h}{L} w_{1}+\left(\frac{h}{L}\right)^{2} w_{2}+H \cdot O \cdot T
$$


A general solution to this problem is obtained by a finite-element discretization of the cross section. The firstorder solution results in ${ }^{14}$

$$
w\left(x_{1}, x_{2}, x_{3}\right)=w_{\gamma}\left(x_{2}, x_{3}\right) \hat{\gamma}\left(x_{1}\right)+w_{\kappa}\left(x_{2}, x_{3}\right) \hat{\kappa}\left(x_{1}\right)+w_{q_{n}}\left(x_{2}, x_{3}\right) \hat{q}_{n}\left(x_{1}\right)+w_{q_{n}^{\prime}}\left(x_{2}, x_{3}\right) \hat{q}_{n}^{\prime}\left(x_{1}\right)+\text { H.O.T. }
$$

Therefore, as a result of this linear optimal problem one obtains a matrix of first-order warping influence coefficients, $\left[\begin{array}{llll}w_{\gamma} & w_{\kappa} & w_{q_{n}} & w_{q_{n}^{\prime}}\end{array}\right]$. The strain energy density can be explicitly integrated at each cross section to define the strain energy per unit length of the beam as

$$
\mathcal{U}=\int_{A(x)} u d A=\frac{1}{2}\left\{\begin{array}{llll}
\gamma^{T} & \kappa^{T} & q^{T} & q^{\prime T}
\end{array}\right\}[S]\left\{\begin{array}{l}
\gamma \\
\kappa \\
q \\
q^{\prime}
\end{array}\right\}+\text { H.O.T. }
$$

where the constant matrix $[S]$ is the first-order asymptotic approximation to the stiffness matrix. Higher-order approximations were presented in Ref. 14. To complete the solution of the cross-sectional problem, the kinetic energy density of Eq. (12) needs to be evaluated using the decomposition of the velocity field given by Eq. (17). With the assumption of negligible contributions of $\dot{\mathbf{w}}$ and $\boldsymbol{\Omega} \times\left(\boldsymbol{\Psi}_{\mathrm{n}} q_{n}+\mathbf{w}\right)$, this task is straightforward, and defines the kinetic energy per unit length as

$$
\mathcal{K}=\int_{A} \kappa d A=\frac{1}{2}\left\{\begin{array}{lll}
V_{B}^{T} & \Omega_{B}^{T} & \dot{q}_{n}^{T}
\end{array}\right\}[M]\left\{\begin{array}{c}
V_{B} \\
\Omega_{B} \\
\dot{q}
\end{array}\right\},
$$

where the constant matrix $[\mathrm{M}]$ is the inertia matrix for the cross section.

\section{One-dimensional analysis at the reference line}

The small-scale cross-sectional problem has defined the homogenization constants for the cross section, and the problem has been effectively reduced to the analysis of the evolution of averaged variables along the reference line. This 1-D long-scale problem is now set up in a continuous segment of arc length $l$ of the reference line, which will be referred as a structural member. The solution procedure follows the one developed by Hodges ${ }^{21}$. From Eq. (22) and (23), the left-hand side terms in Eq. (11) can be integrated at each cross section, as

$$
\overline{\delta \pi} \equiv \int_{0}^{l}[\delta(\mathcal{K}-\mathcal{U})+\overline{\delta \mathcal{W}}] d x,
$$

where $\overline{\delta \pi}$ is the virtual total potential and $\overline{\delta W}$ is the virtual work per unit length of the applied loads, which is decomposed in the virtual work per unit length of the structural damping forces and of the applied external forces, $\overline{\delta W_{d}}$ and $\overline{\delta W_{e}}$, respectively, as

$$
\overline{\delta W}=\overline{\delta W_{d}}+\overline{\delta W_{e}} .
$$

In order to evaluate the different terms in Eq. (24), one needs to select first an irreducible (as defined in Ref. 27) set of independent variables to obtain variations of the functionals. Six independent variables (three displacements, $u$, and three rotations, $\varphi$ of the reference frame $a$ ) determine the rigid body motion of the member. Additional independent variables account for the deformations of the flexible structure: the set of displacements and rotations along the reference line, as well as the amplitudes of the finite-section modes. 


\section{Strain energy per unit length}

In Eq. (22) the strain energy per unit length was approximated as a quadratic functional of the form $V=V\left(\gamma(x), \kappa(x), q(x), q^{\prime}(x)\right)$. The partial derivatives of the strain energy are identified as section stress resultants,

$$
F_{B}=\left(\frac{\partial v}{\partial \gamma}\right)^{T}, M_{B}=\left(\frac{\partial v}{\partial \kappa}\right)^{T}, Q_{s_{0}}=\left(\frac{\partial v}{\partial q}\right)^{T}, Q_{s_{1}}=\left(\frac{\partial v}{\partial q^{\prime}}\right)^{T} .
$$

where $F_{B}$ and $M_{B}$ can be identified as the internal force and moment column vectors, whereas the $Q_{s}$ terms are the conjugate forces corresponding to the deformation of the structure in the finite-section modes. The variation of the internal energy density is then given by

$$
\delta \mathcal{U}=\delta \gamma^{T} F_{B}+\delta \kappa^{T} M_{B}+\delta q^{T} Q_{s_{0}}+\delta q^{\prime T} Q_{s_{1}} .
$$

Variations in beam strain measures need to be written as functions of the virtual displacements and rotations. The column vector of virtual rotations from frame $a$ to frame $B$, expressed in frame $a, \overline{\delta \Phi}_{a}$, is defined as

$$
\tilde{\delta \Phi}_{a}=\delta C^{a B} C^{B a}=-C^{a B} \delta C^{B a} .
$$

One can establish the virtual strain-displacement relations from Eq. (16). They are

$$
\begin{gathered}
\delta \gamma=C^{B a}\left(\frac{d}{d x} \delta R_{a}+\tilde{R}_{a}^{\prime} \overline{\delta \Phi}_{a}\right)=C^{B a} \frac{d}{d x} \delta R_{a}+\left(\tilde{\gamma}+\tilde{e}_{1}\right) C^{B a} \overline{\delta \Phi}_{a}, \\
\delta \kappa=C^{B a} \frac{d}{d x} \overline{\delta \Phi}_{a}, \\
\delta q^{\prime}=\frac{d}{d x} \delta q .
\end{gathered}
$$

\section{Kinetic energy per unit length}

Homogenization of the cross-sectional inertia properties in Eq. (23) approximates the kinetic energy per unit length as a bilinear functional of the form $\mathcal{K}=\mathcal{K}\left(V_{B}(x), \Omega_{B}(x), \dot{q}(x)\right)$. The partial derivatives of the kinetic energy define the section momentum resultants,

$$
P_{B}=\left(\frac{\partial \mathcal{K}}{\partial V_{B}}\right)^{T}, H_{B}=\left(\frac{\partial \mathcal{K}}{\partial \Omega_{B}}\right)^{T}, Q_{t}=\left(\frac{\partial \mathcal{K}}{\partial \dot{q}}\right)^{T},
$$

where $P_{B}$ and $H_{B}$ can be identified as the linear and angular momentum column vectors, whereas $Q_{t}$ are the generalized inertia momenta associated with the finite-section modes. From the previous definitions, the variation of the kinetic energy density is

$$
\delta \mathcal{K}=\delta V_{B}^{T} P_{B}+\delta \Omega_{B}^{T} H_{B}+\delta \dot{q}^{T} Q_{t} .
$$

The virtual quantities in (31) are now expressed as function of the independent variables, as

$$
\begin{gathered}
\delta V_{B}=C^{B a} \delta v_{a}-C^{B a} \tilde{R}_{a} \delta \omega_{a}+C^{B a}\left(\frac{d}{d t} \delta R_{a}+\tilde{\omega}_{a} \delta R_{a}\right)+\tilde{V}_{B} C^{B a} \overline{\delta \Phi}_{a}, \\
\delta \Omega_{B}=C^{B a} \delta \omega_{a}+C^{B a}\left(\frac{d}{d t} \overline{\delta \Phi}_{a}+\tilde{\omega}_{a} \overline{\delta \Phi}_{a}\right), \\
\delta \dot{q}=\frac{d}{d t} \delta q,
\end{gathered}
$$


where the first two equations were obtained taking variations in Eq. (18). The variations of the rigid-body virtual velocities and displacements in the member frame can be expressed as

$$
\begin{aligned}
& \delta v_{a}=\delta \dot{u}_{a}+\tilde{\omega}_{a} \delta u_{a}, \\
& \delta \omega_{a}=\overline{\delta \dot{\varphi}}_{a}+\tilde{\omega}_{a} \overline{\delta \varphi}_{a} .
\end{aligned}
$$

Virtual work per unit length of the external forces

Consider the vector $\mu$ of distributed forces per unit volume applied on the 3-D solid. The virtual work per unit length of the reference line produced by this force is given by multiplying it by the virtual displacement with respect to an inertial frame (the global frame $o$ ), as

$$
\overline{\delta W_{e}}=\int_{A(x)} \boldsymbol{\mu} \cdot(\boldsymbol{\delta} \mathbf{X}+\boldsymbol{\delta} \mathbf{u}) d A
$$

The position vector $\mathbf{X}$ is given in Eq. (7) as function of the deformation of the reference line and the crosssectional warping. The latter can be approximated by the warping influence coefficients obtained in Eq. (21) to give

$$
X_{o}=C^{o a} R_{a}+C^{o B}\left(\xi+\Psi q+w_{\chi} \chi\right)
$$

where $\xi^{T}=\left\{\begin{array}{lll}0 & x_{2} & x_{3}\end{array}\right\}$ contains the cross-sectional coordinates, and $\chi$ includes the elastic degrees of freedom, as

$$
\chi^{T}=\left\{\begin{array}{llll}
\gamma^{T} & \kappa^{T} & q^{T} & q^{\prime T}
\end{array}\right\}
$$

The corresponding virtual displacements are

$$
\delta X_{o}=-C^{o a} \tilde{X}_{a} \overline{\delta \varphi}_{a}+C^{o a} \delta R_{a}-C^{o B}\left(\tilde{\xi}+\tilde{\Psi} q+\tilde{w}_{,} \chi\right) C^{B a} \overline{\delta \Phi}_{a}+C^{o B}\left(\Psi \delta q+w_{\chi} \delta \chi\right) .
$$

Substituting this expression in equation (34), one obtains the virtual work per unit length of the applied forces as,

$$
\overline{\delta W_{e}}=\delta u_{a}^{T} C^{a B} f_{0}+{\overline{\delta \varphi_{a}}}_{a}^{T} C^{a B} m_{0}^{*}+\delta R_{a}^{T} C^{a B} f_{0}+\delta R_{a}^{\prime T} C^{a B} f_{1}+\overline{\delta \Phi}_{a}^{T} C^{a B} m_{0}+{\overline{\delta \Phi^{\prime}}}_{a}^{T} C^{a B} m_{1}+\delta q^{T} f_{s_{0}}+\delta q^{\prime T} f_{s_{1}} .
$$

The following applied forces per unit length have been used in this expression:

$$
\begin{array}{cc}
f_{0}=\int_{A(x)} \mu_{B} d A, & f_{1}=\int_{A(x)} w_{\gamma}^{T} \mu_{B} d A, \\
f_{s_{0}}=\int_{A(x)}\left(\Psi^{T}+w_{q}^{T}\right) \mu_{B} d A, & f_{s_{1}}=\int_{A(x)} w_{q^{\prime}}^{T} \mu_{B} d A, \\
m_{0}^{*}=\int_{A(x)} C^{B a} \tilde{X}_{a} C^{a B} \mu_{B} d A, & m_{1}=\int_{A(x)} w_{\kappa}^{T} \mu_{B} d A . \\
m_{0}=m_{0}^{*}-C^{B a} \tilde{R}_{a} C^{a B} f_{0}, &
\end{array}
$$

where $\mu_{B}$ is the projection of the distributed force $\boldsymbol{\mu}$ onto the deformed reference, $B$. From Eqs. (35) and (39), the virtual work of the external forces depends on the actual deformation through the cross-sectional warping field. The applied work also includes the contribution from the resultant applied forces per unit length conjugated of the finitesection modes $f_{s_{0}}$ and its longitudinal derivatives, $f_{s_{1}}$.

If cross sections are rigid, and the motion of the member reference frame $(a)$ is prescribed, that is, $\delta u_{a}=\overline{\delta \varphi_{a}}=0$, then the only non-zero terms in Eq. (39) are the classical beam forces and moments, 


$$
f_{0, c l}=f_{0}=\int \mu_{B} d A, \quad m_{0, c l}=\int \tilde{\xi} \mu_{B} d A
$$

If, in addition to those, finite-section modes are added as a first approximation to the warping field, and no other warping effects are included, then an additional force term must be considered, given by

$$
f_{s_{0}, c l}=\int \Psi^{T} \mu_{B} d A
$$

\section{Virtual work per unit length of the structural damping forces}

Structural damping is modeled here by forces/moments per unit length proportional to the rate of change of the generalized internal forces (expressed in their components in the deformed frame) at each cross section, that is

$$
\left\{\begin{array}{l}
f_{d 0 B} \\
m_{d 0 B} \\
f_{s d_{0}} \\
f_{s d_{1}}
\end{array}\right\}=-g \frac{d}{d t}\left\{\begin{array}{l}
F_{B} \\
M_{B} \\
Q_{s_{0}} \\
Q_{s_{1}}
\end{array}\right\}=-\left[\begin{array}{cccc}
g_{F} & 0 & 0 & 0 \\
0 & g_{M} & 0 & 0 \\
0 & 0 & g_{s_{0}} & 0 \\
0 & 0 & 0 & g_{s_{1}}
\end{array}\right] \frac{d}{d t}\left\{\begin{array}{c}
F_{B} \\
M_{B} \\
Q_{s_{0}} \\
Q_{s_{1}}
\end{array}\right\},
$$

where $g$ is a diagonal matrix of non-negative structural damping coefficients. The virtual work of these forces is given by

$$
\overline{\delta W_{d}}=-\delta R_{a}^{T} C^{a B} g_{F} \dot{F}_{B}-\overline{\delta \Phi}_{a}^{T} C^{a B} g_{M} \dot{M}_{B}-\delta q^{T} g_{s_{0}} \dot{Q}_{s_{0}}-\delta q^{\prime T} g_{s_{1}} \dot{Q}_{s_{1}}
$$

\section{Intrinsic equations in the member frame}

The strain energy (27), kinetic energy (31), and virtual work (38) and (43) are substituted into the expression of the total potential per unit length (24). Through integration by parts in both time and space, one obtains the geometrically-nonlinear intrinsic equilibrium equations at the reference line. In strong form, they are written as

$$
\begin{gathered}
\left(\frac{d}{d t}+\tilde{\Omega}_{B}\right) P_{B}=\left(\frac{d}{d x}+\tilde{K}_{B}\right)\left(F_{B}-f_{1}\right)-g_{F} \frac{d}{d t} F_{B}+f_{0}, \\
\left(\frac{d}{d t}+\tilde{\Omega}_{B}\right) H_{B}+\tilde{V}_{B} P_{B}=\left(\frac{d}{d x}+\tilde{K}_{B}\right)\left(M_{B}-m_{1}\right)+\left(\tilde{e}_{1}+\tilde{\gamma}\right) F_{B}-g_{M} \frac{d}{d t} M_{B}+m_{0}, \\
\frac{d}{d t} Q_{t}=\frac{d}{d x}\left(Q_{s_{1}}+g_{s_{1}} \frac{d}{d t} Q_{s_{1}}-f_{s_{1}}\right)-\left(Q_{s_{0}}+g_{s_{0}} \frac{d}{d t} Q_{s_{0}}-f_{s_{0}}\right) .
\end{gathered}
$$

If the rigid-body motion of the member is unconstrained, the system is completed by the free-body equations given in the member frame

$$
\begin{aligned}
& \left(\frac{d}{d t}+\tilde{\omega}_{a}\right) p_{a}=\int_{0}^{l} C^{a B} f_{0} d x, \\
& \left(\frac{d}{d t}+\tilde{\omega}_{a}\right) h_{a}=\int_{0}^{l} C^{a B} m_{0}^{*} d x .
\end{aligned}
$$

where two new aggregate vector magnitudes were defined to describe the free-body motion of the member: $\mathbf{p}$, the member translational momentum, and $\mathbf{h}$, the member angular momentum about the origin of the member reference frame, $a$. Their components in the $a$ frame are given by 


$$
\begin{gathered}
p_{a}=\int_{0}^{l} C^{a B} P_{B} d x, \\
h_{a}=\int_{0}^{l}\left(C^{a B} H_{B}+\tilde{R}_{a} C^{a B} P_{B}\right) d x .
\end{gathered}
$$

Eqs. (45) are the expression for an elastic system of the Euler-Lagrange equations for the dynamics of the rigid body. Note that both Eqs. (44) and (45) are given in their intrinsic form, that is, the local elastic equilibrium Eqs. (44) are given in the local deformed frame, $B$, and the global dynamic equilibrium Eqs. (45) are given in the member frame, $a$ (a body-fixed representation, if frame $a$ is forced to move with the member). Three different extensions can be identified in this formulation when compared from the original intrinsic theory of moving beams of Hodges ${ }^{21}$. First, the motion of the reference frame is brought into the system by including Eq. (45). Second, the addition of the finite-section deformation modes into the 1-D representation of the structure brings an additional differential equation for each mode into the final system, Eq. (44). And third, a more general definition of the forcing terms in the 1-D equations is introduced in Eq. (39) by using the actual structural deformation (defined by a set of warping influence coefficients in the cross-sectional analysis) in the computation of the virtual work of the external forces.

\section{Mixed-Form Finite-Element Solution}

A numerical solution is proposed based on a mixed (or hybrid) form of the equations on the reference line, as it was done by Hodges et $a l^{22}$. For that purpose, the weak form of the equilibrium equations (44) and (45) was augmented imposing the kinematical relations (16) and (18) through Lagrange multipliers. In the present implementation, it is assumed that the motion of the member reference frame $(a)$ is prescribed. Rotations are parameterized using Rodrigues parameters between the member reference frame $(a)$ and the deformed frame $(B)$, $\theta_{a}^{B}$. The direct and inverse relations between rotation matrix and Rodrigues parameters are

$$
\tilde{\theta}_{a}^{B}=-2\left(1+\operatorname{tr}\left(C^{B a}\right)\right)^{-1}\left(C^{B a}-C^{a B}\right), \text { and } \quad C^{B a}=I+\left(1+\frac{1}{4}\left(\theta_{a}^{B}\right)^{T} \theta_{a}^{B}\right)^{-1}\left(-\tilde{\theta}_{a}^{B}+\frac{1}{2}\left(\tilde{\theta}_{a}^{B}\right)^{2}\right) .
$$

Note that this parameterization includes any initial twist and curvature of the reference line. The mixed form of the member equations can be written as

$$
\begin{aligned}
& \int_{t_{1}}^{t_{2}} \overline{\delta \pi^{*}} d t=\int_{t_{1}}^{t_{2}}\left\{\int _ { 0 } ^ { L } \left\{\delta R_{a}^{T} C^{a B}\left[\left(\frac{d}{d t}+\tilde{\Omega}_{B}\right) P_{B}+g_{F} \frac{d}{d t} F_{B}-f_{0}\right]+\delta R_{a}^{\prime T} C^{a B}\left[F_{B}-f_{1}\right]+\right.\right. \\
&+ \delta F_{a}^{T} C^{a B}\left[e_{1}+\gamma\right]+\delta F_{a}^{\prime T} R_{a}-\delta P_{a}^{T}\left[C^{a B} V_{B}-\left(\frac{d}{d t}+\tilde{\omega}_{a}\right) R_{a}-v_{a}\right]+ \\
&+\overline{\delta \Phi}_{a}^{T} C^{a B}\left[\left(\frac{d}{d t}+\tilde{\Omega}_{B}\right) H_{B}+\tilde{V}_{B} P_{B}+g_{M} \frac{d}{d t} M_{B}-m_{0}\right]+{\overline{\delta \Phi^{\prime}}}_{a}^{T} C^{a B}\left[M_{B}-m_{1}\right]+ \\
&+\delta M_{a}^{T} \rho^{-1}\left(\theta_{a}^{B}\right) \cdot\left(\kappa+k_{b}\right)+\delta M_{a}^{\prime T} \theta_{a}^{B}-\delta H_{a}^{T}\left[\rho^{-1}\left(\theta_{a}^{B}\right)\left(\Omega_{B}-C^{B a} \omega_{a}\right)-\dot{\theta}_{a}^{B}\right]+ \\
&\left.+\delta q\left(\frac{d}{d t} Q_{t}+Q_{s_{0}}+g_{s_{0}} \dot{Q}_{s_{0}}-f_{s_{0}}\right)+\delta q^{\prime}\left(Q_{s_{1}}+g_{s_{1}} \dot{Q}_{s_{1}}-f_{s_{1}}\right)+\delta Q_{s_{1}} q^{\prime}+\delta Q_{s_{1}}^{\prime} q-\delta Q_{t}\left(\dot{q}-\frac{d}{d t} q\right)\right\} d x+ \\
&\left.-\left[\delta R_{a}^{T} \hat{F}_{a}+\overline{\delta \Phi}_{a}^{T} \hat{M}_{a}+\delta q \hat{Q}_{s_{1}}+\delta F_{a}^{T} \hat{R}_{a}+\delta M_{a}^{T} \hat{\theta}_{a}^{B}+\delta Q_{s_{1}} \hat{q}\right]_{0}^{l}\right\} d t= \\
&=-\int_{0}^{l}\left[\delta R_{a}^{T}\left(\hat{P}_{a}-P_{a}\right)+\overline{\delta \Phi}_{a}^{T}\left(\hat{H}_{a}-H_{a}\right)+\delta q^{T}\left(\hat{Q}_{t}-Q_{t}\right)\right]_{t_{1}}^{t_{2}} d x,
\end{aligned}
$$

where the symbol $\hat{\bullet}$ refers to prescribed values (boundary values at the ends of either the time or the space domain) and the $3 \times 3$ matrix $\rho(\theta)$ is the rotational (or tangential) operator, whose inverse is defined as

$$
\rho^{-1}(\theta)=I+\frac{1}{2} \tilde{\theta}+\frac{1}{4} \theta \theta^{T} .
$$

Eq. (48) is an appropriate starting point to obtain a finite-element (spatial) discretization of the 1-D dynamic equations. The discretization is defined in each member; then, members are assembled using the boundary and/or joint conditions at both ends. If free-body motions are unconstrained, the problem is completed by adding the rigid- 
body equations from (45). The dependency with time is left explicitly and the original system of partial-differential equations is converted into a set of ordinary-differential/algebraic equations in time domain. The generalized virtual potential is discretized in $N$ elements at a given time as

$$
\overline{\delta \pi^{*}}=\sum_{e=1}^{N} \overline{\delta \pi_{e}^{*}} .
$$

The length of the $e$-th element is $\Delta l_{e}$, and is bounded by nodes $e$ and $e+1$. The mixed formulation allows the selection of the following shape functions in the element,

$$
\begin{gathered}
\left\{\begin{array}{c}
R_{a}(x) \\
\theta_{a}^{B}(x) \\
q(x)
\end{array}\right\}=\left\{\begin{array}{l}
R_{e} \\
\theta_{e} \\
q_{e}
\end{array}\right\}, \quad\left\{\begin{array}{c}
\delta R_{a}(x) \\
\delta \Phi_{a}(x) \\
\delta q(x)
\end{array}\right\}=(1-\zeta)\left\{\begin{array}{l}
\frac{\delta R_{e}}{\delta \Phi_{e}} \\
\delta q_{e}
\end{array}\right\}+\zeta\left\{\begin{array}{l}
\delta R_{e+1} \\
\delta \Phi_{e+1} \\
\delta q_{e+1}
\end{array}\right\}, \\
\left\{\begin{array}{c}
F_{B}(x) \\
M_{B}(x) \\
Q_{s_{1}}(x)
\end{array}\right\}=\left\{\begin{array}{l}
F_{e} \\
M_{e} \\
Q_{s e}
\end{array}\right\},\left\{\begin{array}{l}
\delta F_{a}(x) \\
\delta M_{a}(x) \\
\delta Q_{s_{1}}(x)
\end{array}\right\}=(1-\zeta)\left\{\begin{array}{l}
\delta F_{e} \\
\delta M_{e} \\
\delta Q_{s e}
\end{array}\right\}+\zeta\left\{\begin{array}{l}
\delta F_{e+1} \\
\delta M_{e+1} \\
\delta Q_{s e+1}
\end{array}\right\}, \\
\left\{\begin{array}{l}
P_{B}(x) \\
H_{B}(x) \\
Q_{t}(x)
\end{array}\right\}=\left\{\begin{array}{l}
P_{e} \\
H_{e} \\
Q_{t e}
\end{array}\right\},\left\{\begin{array}{l}
\delta P_{a}(x) \\
\delta H_{a}(x) \\
\delta Q_{t}(x)
\end{array}\right\}=\left\{\begin{array}{l}
\delta P_{e} \\
\delta H_{e} \\
\delta Q_{t e}
\end{array}\right\},
\end{gathered}
$$

with

$$
x \in\left[x_{e}, x_{e+1}\right] \text {, and } \zeta=\Delta l_{e}^{-1}\left(x-x_{e}\right) .
$$

Therefore, the continuous virtual quantities within each element are approximated by linear variations between the discrete nodal values in displacements and forces, and constant values for the virtual momenta. The problem unknowns are approximated as constant values within each element. This defines a $3 \times\left(6+N_{q}\right)$ element state vector, where $N_{q}$ is the number of finite-section modes, as

$$
\mathrm{X}_{e}^{T}(t)=\left[\begin{array}{lllllllll}
R_{e}^{T} & \theta_{e}^{T} & q_{e}^{T} & F_{e}^{T} & M_{e}^{T} & Q_{s e}^{T} & P_{e}^{T} & H_{e}^{T} & Q_{t e}^{T}
\end{array}\right]
$$

and a member state vector as

$$
\mathrm{X}^{T}(t)=\left[\begin{array}{lll}
\mathrm{X}_{1}^{T} & \ldots & \mathrm{X}_{N}^{T}
\end{array}\right]
$$

The boundary values of displacements and forces at $x=0$ and $x=l$ defined additional unknowns in the problem. Their components in the member reference frame $(a)$ define the member boundary state vector as

$$
\hat{\mathrm{X}}_{x=x_{0}}^{T}(t)=\left[\begin{array}{llllll}
\hat{R}_{x=x_{0}}^{T} & \hat{\theta}_{x=x_{0}}^{T} & \hat{q}_{x=x_{0}}^{T} & \hat{F}_{x=x_{0}}^{T} & \hat{M}_{x=x_{0}}^{T} & \hat{Q}_{s_{1}, x=x_{0}}^{T}
\end{array}\right] .
$$

The total number of unknowns in the discretization of the structural member is then $U=(3 \times N+4) \times\left(6+N_{q}\right)$. In general, all of them are functions of time. The former discretization allows the explicit integration in $x$ of equation (50), which can then be written as

$$
\overline{\delta \pi^{*}}=\sum_{e=1}^{N}\left\{\delta \mathrm{X}_{e}^{T}\left(A_{e}^{(1)} \dot{\mathrm{X}}_{e}+\mathrm{S}_{e}^{(1)}-\mathrm{L}_{e}^{(1)}\right)+\delta \mathrm{X}_{e+1}^{T}\left(A_{e}^{(2)} \dot{\mathrm{X}}_{e}+\mathrm{S}_{e}^{(2)}-\mathrm{L}_{e}^{(2)}\right)\right\}=\delta \mathrm{X}_{N+1}^{T} K \hat{\mathrm{X}}_{x=l}-\delta \mathrm{X}_{1}^{T} K \hat{\mathrm{X}}_{x=0} .
$$

where $K$ is defined as the following $\left[3 \times\left(6+N_{q}\right)\right] \times\left[2 \times\left(6+N_{q}\right)\right]$ matrix 


$$
K=\left[\begin{array}{ll}
0 & I \\
I & 0 \\
0 & 0
\end{array}\right]
$$

Imposing arbitrary values to the virtual terms in equation (56), one obtains a set of differential-algebraic equations in time domain for each member:

$$
A(\mathrm{X}) \cdot \dot{\mathrm{X}}=\mathrm{L}\left(\mathrm{X}, \hat{\mathrm{X}}_{x=0}, \hat{\mathrm{X}}_{x=l}\right)-\mathrm{S}\left(\mathrm{X}, \hat{\mathrm{X}}_{x=0}, \hat{\mathrm{X}}_{x=l}\right),
$$

where $A$ is the (singular) inertia matrix operator, $\mathrm{S}$ is the structural column matrix operator, and $\mathrm{L}$ is the load column matrix operator. These equations are complemented by $6+N_{q}$ additional equations on each member end, which can be defined either from boundary conditions (i.e., clamped or free end) or from joint conditions between two or more members.

Three different solution schemes were set up for Eq. (58): a time-domain solution, a steady-state solution, and a linearized vibration analysis. The time-domain solution is performed using an implicit three-point backwards Euler integration scheme with variable time step, which transforms the ordinary differential equations into a set of nonlinear algebraic equations. The steady-state solution is obtained by setting $\dot{\mathrm{X}}=0$. Both the time-domain and the steady-state solutions are then given by algebraic equations, which are solved for each time and load step, respectively, using a Newton-Raphson method with analytical expressions for the Jacobians. The solution is further simplified by using the bandwidth within the member.

Finally, for the linearized vibration solution, the dynamic equations (44) are linearized around a nonlinear steady-state. The complex-domain eigenvalues and eigenvectors from the resulting first-order system are finally obtained using ARPACK ${ }^{28}$.

\section{Numerical Examples}

Three numerical examples are proposed to illustrate a typical application of the present theory. First, the effect of cross-sectional deformations is studied in the natural vibration modes of a simple isotropic thin strip. Results for a 1D model with camber-bending deformations are compared with those of a shell model. The second example is the computation of the local deformations in a cantilever box beam with embedded actuation. Distributed actuation generates local wall deformation, which is captured by the proposed theory by defining finite-section modes on the cross section. Finally, the effect of cross-sectional deformations is studied on the linear vibration characteristics of the same box beam configuration. Results are compared with shell finite-element solutions using MSC.Nastran.

\section{A. Isotropic thin strip}

Consider an isotropic thin strip ( $\left.E=1.0 \mathrm{GPa}, v=0.3, \rho=1000 \mathrm{~kg} / \mathrm{m}^{3}\right)$ of length-to-width ratio $L / h=4$ and crosssectional width-to-thickness ratio $h / t=50$. Thickness was chosen to be $t=2.79 \mathrm{~mm}$. For this configuration the linear vibration modes are computed by a 40-element 1-D model using the present approach, in which camber bending deformations are included by means of a finite-section mode, as

$$
\Psi\left(x_{2}, x_{3}\right)=\left\{\begin{array}{ll}
0 & 0 \\
\left.\frac{2 x_{2}}{h}\right)^{2}-\frac{1}{3}
\end{array}\right\}^{T},
$$

where the reference for the cross-sectional coordinates was selected at the area centroid of the cross section. It can be easily proved that the finite-section mode defined in Eq. (59) satisfies the orthogonality conditions of Eq. (9). Results of the 1-D model are compared with a finite-element shell model with 10×40 elements in MSC.Nastran.

The natural frequencies of the first ten vibration modes of this configuration are included in Table 1 . They are identified as vertical (VB) or lateral (LB) bending modes or twist modes (T). The eigenvectors obtained by both approximations are compared using the Modal Assurance Criteria (MAC) number (i.e., the normalized scalar product of both mode shapes). 
Table 1. Natural frequencies (in Hz) for thin strip.

\begin{tabular}{cccccc}
\hline \hline & \multicolumn{2}{c}{ Shell (2-D) } & \multicolumn{3}{c}{ 1-D with camber } \\
\hline Type & $\#$ & Freq. & $\#$ & Freq. & MAC \\
\hline VB1 & 1 & 1.469 & 1 & 1.457 & 1.000 \\
VB2 & 2 & 9.184 & 2 & 9.152 & 1.000 \\
T1 & 3 & 11.696 & 3 & 11.046 & 0.996 \\
VB3 & 4 & 25.766 & 4 & 25.731 & 1.000 \\
T2 & 5 & 36.081 & 5 & 33.172 & 0.989 \\
VB4 & 6 & 50.642 & 6 & 50.733 & 1.000 \\
T3 & 7 & 63.287 & 7 & 55.401 & 0.971 \\
LB1 & 8 & 69.297 & 8 & 77.163 & 1.000 \\
VB5 & 9 & 83.893 & 10 & 84.555 & 1.000 \\
T4 & 10 & 94.829 & 9 & 77.802 & 0.944 \\
\hline \hline
\end{tabular}

From these results, it should be noted, first, that vertical bending modes compare very well. In particular, the mode shapes obtained by the current 1-D formulation capture the local warping effects, as it can be seen in the $5^{\text {th }}$ bending mode in Figure 2. For the lateral bending and twist modes the 1-D solution gives a larger error, which increases with frequency. For these results to improve the warping constraint at the clamped end should be included into the model. Further extensions of the present theory will address this issue.

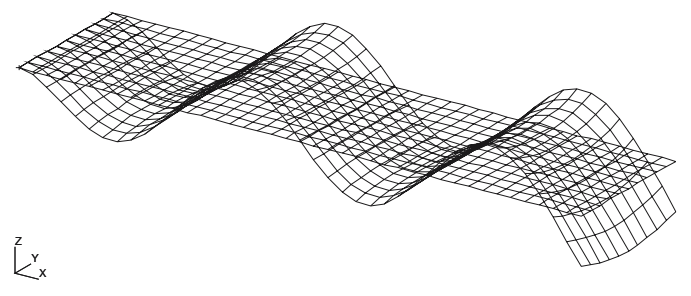

Shell Model

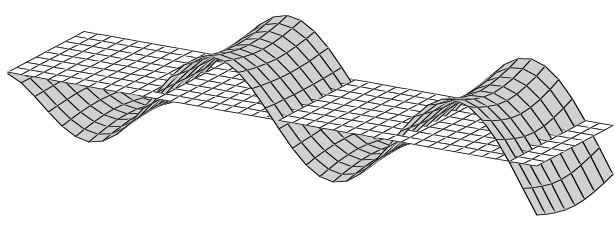

1-D with camber bending

Figure 2. $5^{\text {th }}$ bending mode in shell and 1-D models.

At higher frequencies, some camber-bending-dominated modes start to appear. They would not be captured by a classical (Euler-Bernoulli or Timoshenko) beam model and a finite-section mode for the camber-bending deformation is then required. The frequencies of the first natural vibration modes that can be identified as camberbending-dominated are included in Table 2. The corresponding eigenvectors are shown in Figure 3.

Table 2. Natural frequencies (in Hz) for thin strip. Camber-Bending vibration modes.

\begin{tabular}{ccccc}
\hline \hline \multicolumn{2}{c}{ Shell (2-D) } & \multicolumn{3}{c}{ 1-D with camber } \\
\hline$\#$ & Freq. & $\#$ & Freq. & MAC \\
\hline 17 & 190.23 & 20 & 199.85 & 0.991 \\
18 & 223.63 & 22 & 228.15 & 0.978 \\
21 & 265.79 & 25 & 262.64 & 0.965 \\
\hline \hline
\end{tabular}

As it can be observed, the additional degree of freedom in the 1-D model has captured quite accurately the first vibrations in camber of the thin strip. For shorter plates, higher-order camber deformations could be included by defining additional finite-section modes. 


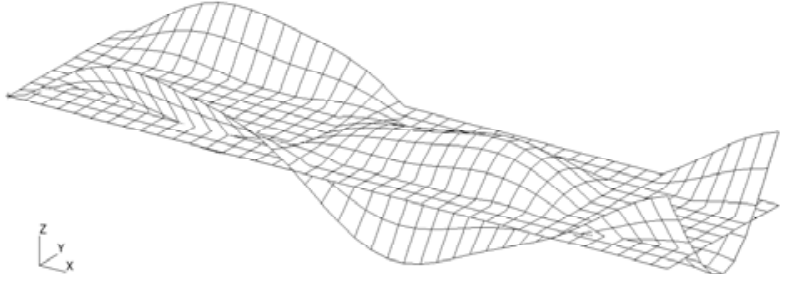

Mode \# 17 - Shell Model

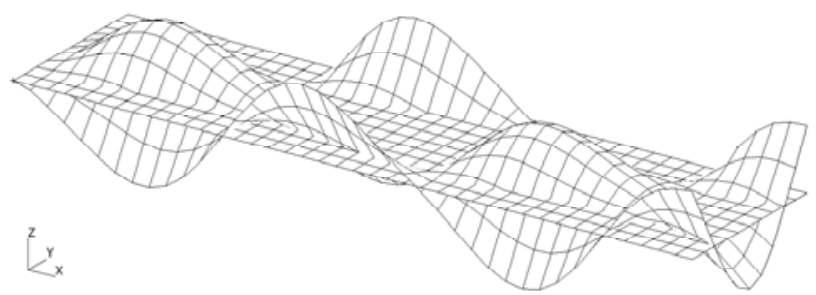

Mode \# 18 - Shell Model

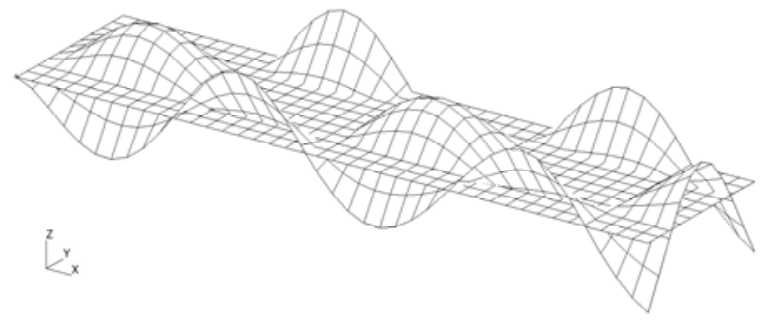

Mode \# 21 - Shell Model

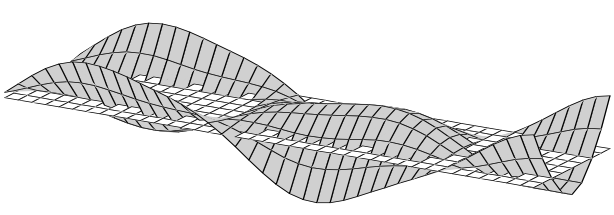

Mode \#20 - 1-D with camber bending

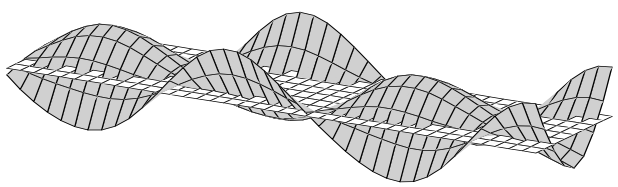

Mode \#22 - 1-D with camber bending

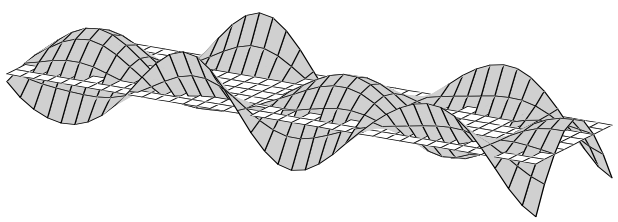

Mode \#25 - 1-D with camber bending

Figure 3. Camber-bending vibration modes for the isotropic thin strip.

\section{B. Static Response of Composite Box Beam with Embedded Actuation}

This second numerical example corresponds to a cantilever composite box-beam with midwall dimensions length $\times$ width $\times$ height equal to $20 a \times 2 a \times a$, and with embedded piezoelectric actuators. Each wall is made of four orthotropic plies of thickness $a / 100$. A dominant dimension can then be identified in the structure and a 1-D model is proposed for its analysis with the reference line passing through the area centroid of the cross sections. The thinwall construction, however, implies the likely appearance of plate-like components in the deformation field, which were found to be particularly important in two situations: 1 ) when the excitation comes from a distributed loading, such as the one generated by embedded piezoelectric actuators; 2) with dynamic excitation at low to moderate frequencies.

Material elastic constants are $E_{33}=E_{22}=E_{11} / 20, G_{12}=G_{13}=E_{11} / 10, G_{23}=G_{12} / 2, v_{12}=v_{13}=0.35$, and $v_{23}=0.4$ (with 1 in the direction of the fiber, 2 in the plane of the plies, and 3 through-the-thickness). All elastic plies are assumed to have piezoelectric properties. For the piezoelectric actuation response, a prescribed through-the-thickness constant electric field $E_{3}$ is assumed on the actuated plies, such that the free-strain deformation of a single ply is given by $d_{3,11} E_{3}=\Gamma_{11}^{o}$ in the direction of the fiber, and with $d_{3,22}=-d_{3,11} / 3$ in the transverse direction. This characterization of the electric field corresponds to the usual assumption for the analysis of piezoelectric actuators in structural applications $^{29}$.

Two different lay-ups with several actuation architectures are studied and are included in Table 3, where plies are numbered inside out and positive material orientation angle is defined in the $x_{1}-x_{2}$ local plane of Figure 4 . 
Table 3. Lay-up and electric actuation for box-beam.

\begin{tabular}{ccc}
\hline \hline Case & Elastic ply-up & Electric actuation \\
\hline S1 & & $(0,1) \downarrow ;(0 /+/ 0 /-) \Rightarrow \Leftarrow$ \\
S2 & $(0 /+/+/ 0) \Uparrow \downarrow \Rightarrow \Leftarrow$ \\
S3 & $\left(45_{2} /-45_{2}\right) \Uparrow \Rightarrow ;\left(-45_{2} / 45_{2}\right) \downarrow \Leftarrow$ & $(0 /+/+/ 0) \Uparrow \Leftarrow ;(0 /-/-/ 0) \downarrow \Rightarrow$ \\
S4 & & $\left(0_{4}\right) \Uparrow \downarrow ;(-/ 0 / 0 /+) \Rightarrow ;(+/ 0 / 0 /-) \Leftarrow$ \\
\hline A1 & & $(+/+/+/+) \Rightarrow ;(-/ /-/-) \Leftarrow$ \\
A2 & $\left(45 /-45_{2} / 45\right) \Uparrow \downarrow \Rightarrow \Leftarrow$ & $(+/-/-/+) \Rightarrow \Leftarrow$ \\
A3 & & $(-/ 0 / 0 /+) \Rightarrow \Leftarrow$ \\
A4 & & $(-/ 0 / 0 /+) \Rightarrow ;(+/ 0 / 0 /-) \Leftarrow$ \\
\hline \hline
\end{tabular}

$\Uparrow$ upper, $\Downarrow$ bottom, $\Rightarrow$ right, $\Leftarrow$ left wall; plies numbered inside out

In the numerical analysis, results of the present 1-D model are compared with a detailed (2-D) shell finiteelement model using composite element properties with a similar longitudinal discretization.

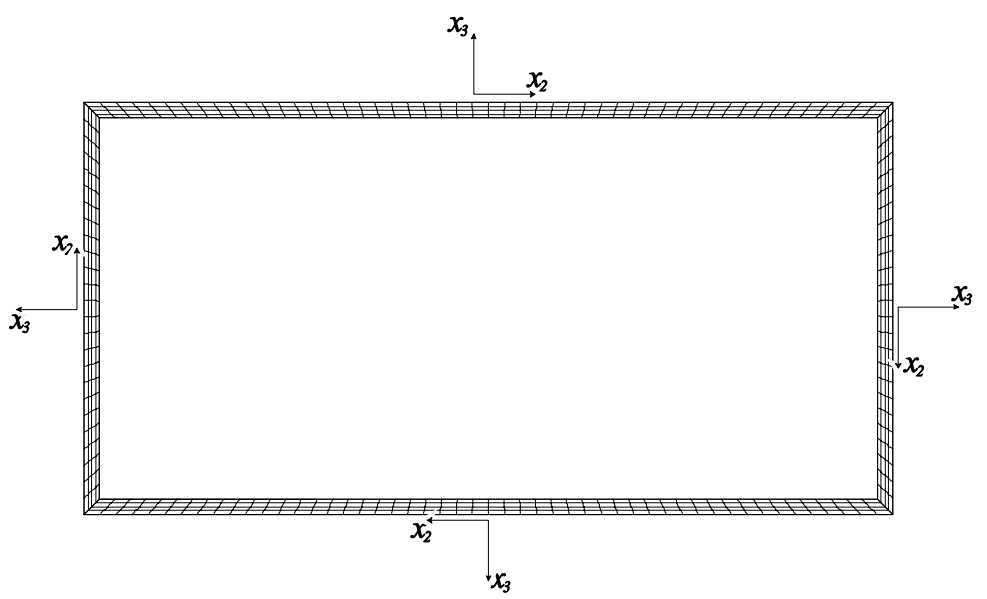

Figure 4. Cross-sectional finite-element discretization showing local coordinate frames.

Consider first a classical 1-D description for the box beam, such that its state is just defined by displacements and rotations of the reference line. The strain energy of Eq. (26) is then defined by the six (BEST: BendingExtension-Shear-Twist) elastic states in Timoshenko beam theory. Note that, although in thin-wall beams shear relief effects are quite small, transverse shear effects are still necessary to account for possible net shear actuation forces. The resulting static displacements $\left(u_{i}\right)$ and rotations $\left(\theta_{i}\right)$ at the beam midpoint $\left(x_{1}=10 a\right)$ for the different actuator configurations defined in Table 3 are shown in Table 4. Displacements obtained by the 1-D beam model are compared with the kinematical interpolation of the shell displacements field at the reference line.

Table 4. Displacements and rotations at $\mathbf{x}=\mathbf{1 0 a}$ with static actuation $\left(\mathbf{a}=\mathbf{0 . 5}, \mathbf{E}_{11}=10^{7}, \Gamma_{11}^{o}=300 \mu \varepsilon\right)$.

\begin{tabular}{lccccccc}
\hline \hline Case & Model & $u_{1}(\mathrm{~mm})$ & $u_{2}(\mathrm{~mm})$ & $u_{3}(\mathrm{~mm})$ & $\theta_{1}(\mathrm{deg})$ & $\theta_{2}$ (deg) & $\theta_{3}$ (deg) \\
\hline S1 & $2-D / 1-D$ & - & $0.02 / 0.03$ & $-1.42 /-1.39$ & - & - & - \\
S2 & $2-D / 1-D$ & $0.70 / 0.69$ & - & - & - & - & - \\
S3 & $2-D / 1-D$ & - & $2.11 / 2.10$ & $-6.08 /-5.98$ & - & $0.14 / 0.14$ & $0.05 / 0.05$ \\
S4 & $2-D / 1-D$ & - & $0.08 /-0.04$ & $0.04 / 0.01$ & $0.07 / 0.07$ & - & - \\
A1 & $2-D / 1-D$ & - & $-4.21 /-4.15$ & - & - & - & $-0.10 /-0.10$ \\
A2 & $2-D / 1-D$ & - & - & - & $-0.17 /-0.17$ & - & - \\
A3 & $2-D / 1-D$ & - & - & - & - & - & - \\
A4 & 2-D/1-D & - & $0.09 /-0.11$ & - & - & - & - \\
\hline \hline
\end{tabular}


In most situations the 3-D displacement field induced by the embedded actuators can be easily identified as beam deformations: that is the case for configurations S1 (transverse shear), S2 (extension), S3 (twist/bending), A1 (bending), and A2 (twist). However, in some cases local wall deformations are important and Timoshenko's (BEST) description is not able to capture even the average motion of the structure in all situations. To solve this, the 1-D model is expanded with a set of finite-section modes, as defined in Eq. (7), which account for the local deformations of the beam walls. For the box beam under consideration, finite-section modes are defined by harmonic functions at the walls, $\psi_{q}=\cos \left(k \pi x_{\alpha} / L_{\alpha}\right)$ ( $L_{\alpha}$ is the wall length and $k=1$, 2. Note that the actual amplitude of $\psi_{q}$ does not affect the results). This set of modes defines a unique expansion of the cross-sectional warping field. Only the six modes included in Figure 5 are considered in the final 1-D model, which will therefore have twelve independent variables (three translations, three rotations and six amplitudes of the modes along the reference line).

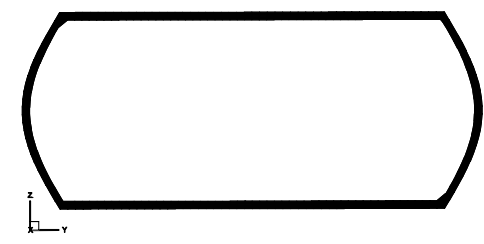

Mode \#121

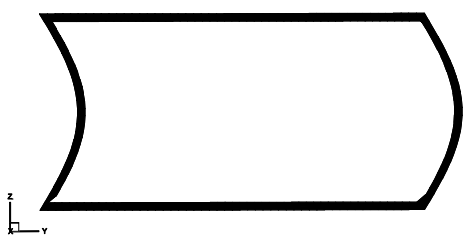

Mode \#123

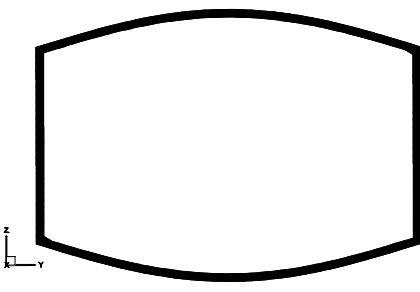

Mode \#122

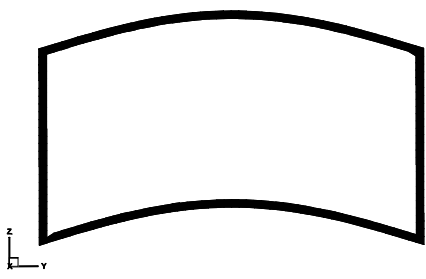

Mode \#124

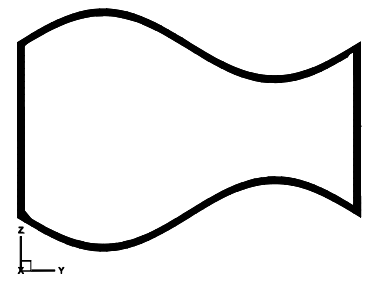

Mode \#222

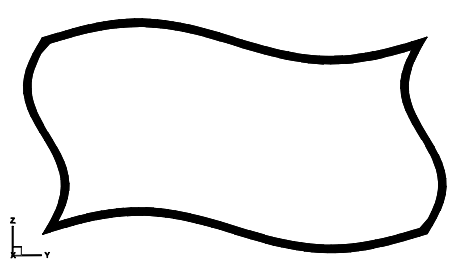

Mode \#241

Figure 5. Finite-section modes for the plate-like deformations at box-beam walls.

It should be remarked that the actual warping field associated to each finite-section mode is obtained through the minimization of the cross-sectional strain energy. The resulting displacement field associated to each finite-section mode is obtained by adding the assumed shape function and the computed warping influence coefficients of Eq. (21). As with the classical deformation measures, the displacement field keeps the averaged value (the integral over the cross section) of the assumed shape functions. Figure 6 includes both the original shape function and the resulting displacement field associated to finite-section modes \#123 and \#124 in a 1-D model that includes the four classical modes and finite-section modes \#121 to \#124.

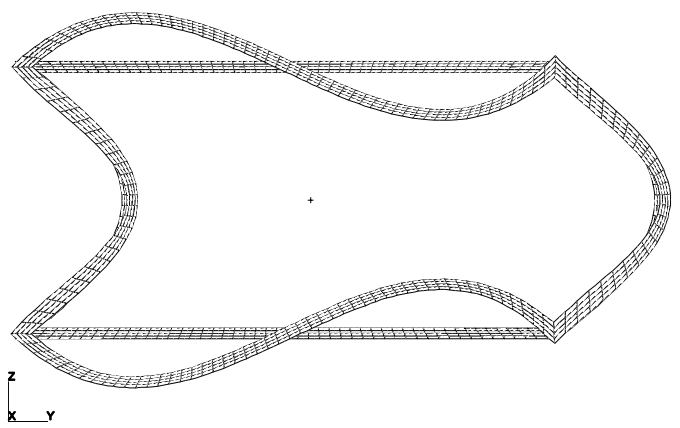

Mode \#123

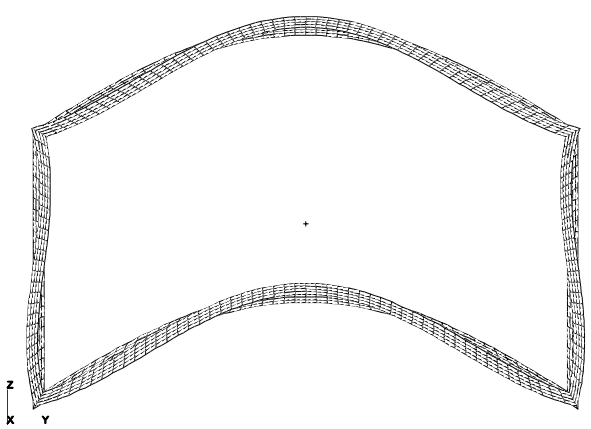

Mode \#124

Figure 6. Prescribed and actual deformation field of finite-section modes. [Difference in the actual deformation between laminates $A$ and $S$ is negligible.]

With that information, the 1-D reduced model is now rebuilt and solved for the different configurations, including the finite-section modes introduced in Figure 5. The 3-D displacement field is constructed from both the 
cross-sectional and longitudinal results using Eq. (35). The resulting displacement field (multiplied by a factor of 100) for several configurations defined in Table 3 is included in Figure 7, where it is compared with the results of built-up shell finite-element models.

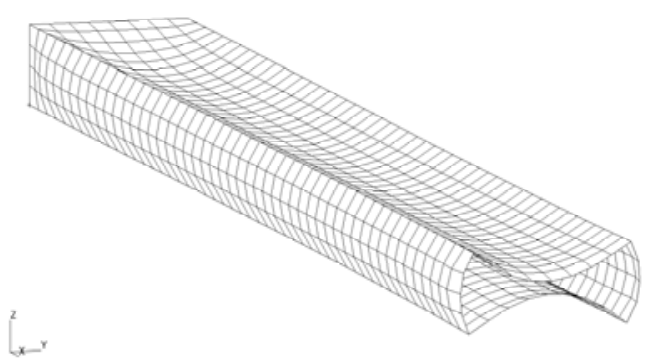

Shell Model. Case A3

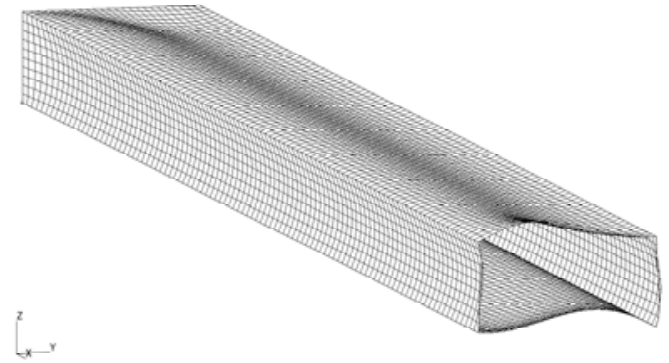

Shell Model. Case A4

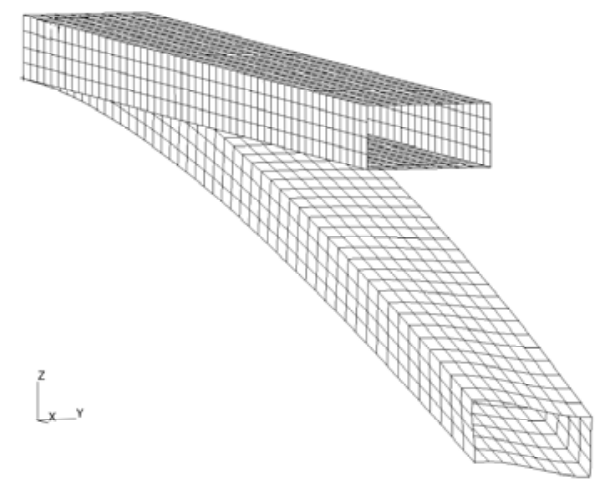

Shell Model. Case S3

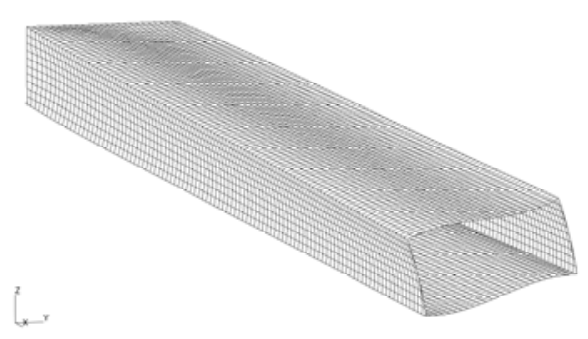

Shell Model. Case S4

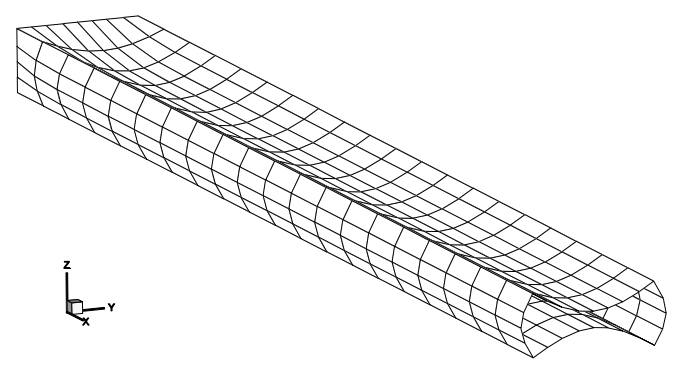

1-D with finite-section. Case A3

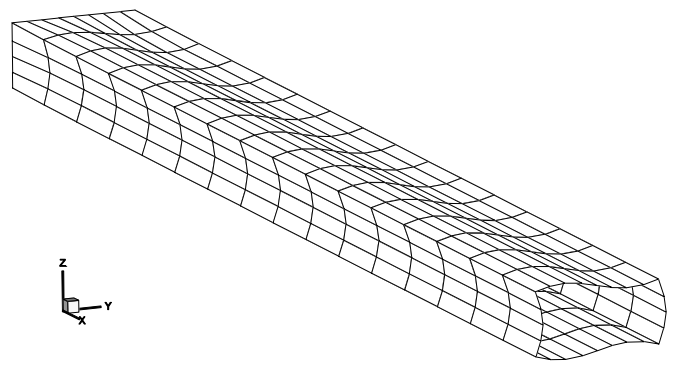

1-D with finite-section. Case A4

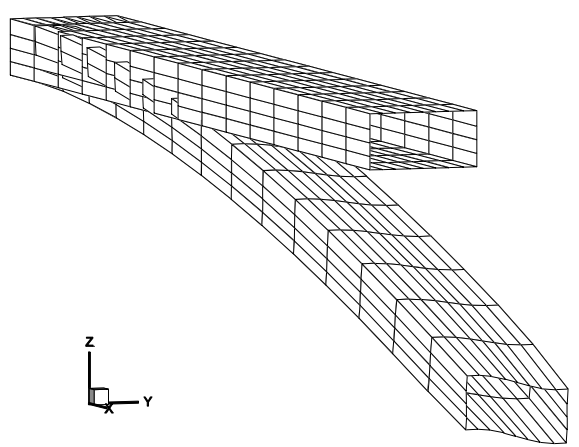

1-D with finite-section. Case S3

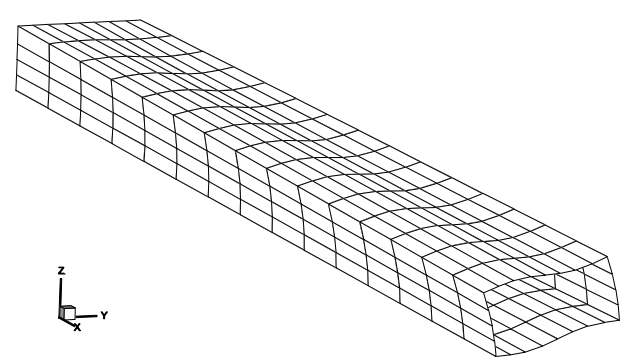

1-D with finite-section. Case S4

Figure 7. Deformed configurations under active static loading $\left(\mathbf{a}=\mathbf{0 . 5}, \mathbf{E}_{\mathbf{1 1}}=\mathbf{1 0}^{7}, \Gamma_{11}^{o}=300 \mu \varepsilon\right.$. Displacements multiplied by 100). 
As it can be seen, the present approach captures both the longitudinal (long-scale) deformation as well as the local (small scale) wall deformations for distributed loading, all in a 1-D solution. Figure 8 includes a direct comparison of the deformed cross section at the center of the beam $(x=10 a)$ for the different actuations on laminate $A$. The correlation between the present method and the shell model is excellent. On the selection of the finite-section modes, it is important to emphasize that the local minimization at the cross-sectional level corrects the given mode, as in Figure 6 to provide a final energy-based 2-D deformation shape. Therefore, details in the original definition of the finite-section modes do not essentially affect the final solution and any general orthogonal expansion, such as the harmonic functions used here, would yield very similar results.
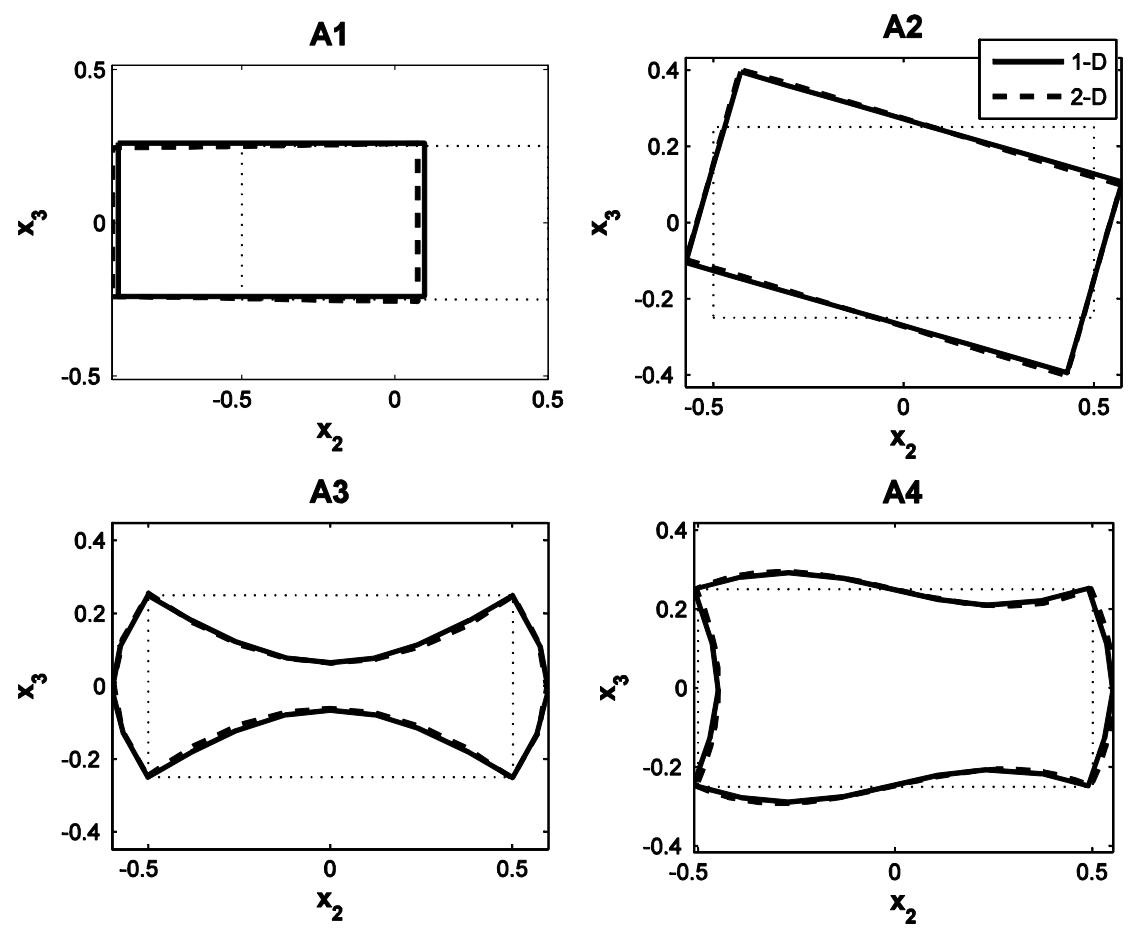

Figure 8. Cross-sectional deformations under static loads at $\mathbf{x}=10 \mathrm{a}$. Laminate $\boldsymbol{A}\left(\mathbf{a}=\mathbf{0 . 5}, \mathbf{E}_{11}=10^{7}, \Gamma_{11}^{o}=300 \mu \varepsilon\right.$. Displacements multiplied by 100).

\section{Linear Vibration Analysis of a Box Beam}

This example investigates the vibration characteristics of the box beam considered above (laminate $A$ ) using the present approach for the solution. Three different 1-D models are considered: 1) a generalized (classical) EulerBernoulli beam model, which includes only four elastic degrees of freedom (extension, twist, and bending in two directions) and six inertial degrees of freedom (three displacements and three rotations); 2) a generalized Timoshenko beam model, which adds the effect of transverse shear strains to the previous elastic description; 3) an expanded model with finite-section modes, where the six additional cross-sectional deformations that were defined in Figure 5 are added to the generalized Timoshenko model. Each finite-section mode adds two elastic states and one inertia state, defined by $q$ and $q^{\prime}$ in Eq. (22) and $\dot{q}$ in Eq. (23), respectively. Finally, results correspond to $\sqrt{E_{11} / \rho a^{2}}=10^{4} s^{-1}$.

For the layup under consideration (laminate $A$ in Table 3), beam bending and twist responses are decoupled when the reference line is located at the area centroid. Therefore, the first vibration modes can be identified as vertical bending, lateral bending, twist, or wall modes.

Table 5 includes the natural frequencies of the first three vertical (flapwise) bending modes of laminate $A$, as obtained by different structural models, and the correlation index among the corresponding mode shapes, defined by the Modal Assurance Criteria (MAC) index. The corresponding mode shapes are included in Figure 9. 
Table 5 Natural frequencies (in Hz) for laminate $A$. Vertical bending modes.

\begin{tabular}{ccccccccccc}
\hline \hline & \multicolumn{1}{c}{ Shell } & \multicolumn{3}{c}{ 1-D Euler-Bernoulli } & \multicolumn{3}{c}{ 1-D Timoshenko } & \multicolumn{2}{c}{ 1-D with finite-section modes } \\
\hline$\#$ & Freq. & $\#$ & Freq. & MAC & $\#$ & Freq. & MAC & $\#$ & Freq. & MAC \\
\hline 1 & 3.34 & 1 & 3.34 & 0.998 & 1 & 3.32 & 0.999 & 1 & 3.33 & 0.998 \\
3 & 19.44 & 3 & 20.82 & 0.986 & 3 & 20.04 & 0.987 & 3 & 19.79 & 0.989 \\
9 & 39.99 & 6 & 57.91 & 0.872 & 6 & 53.24 & 0.878 & 7 & 40.13 & 0.912 \\
\hline \hline
\end{tabular}

As it can be observed, significant improvements can be obtained in the estimation of the modal characteristics by adding additional 1-D degrees of freedom to the model. At higher frequencies, local displacements appear on the beam walls, which are not captured by the 2-D warping influence coefficients of the classical beam modes, i.e., $w_{\gamma}$ and $w_{\kappa}$ in Eq. (35). Adding finite-section modes that explicitly account for the wall deformations provides a much better approximation to the actual vibration characteristics. Note that for these first bending modes, only finitesection mode \#124 in Figure 5 is actually needed to obtain these results, as the contribution of the rest of the modes defined in Figure 5 is negligible.

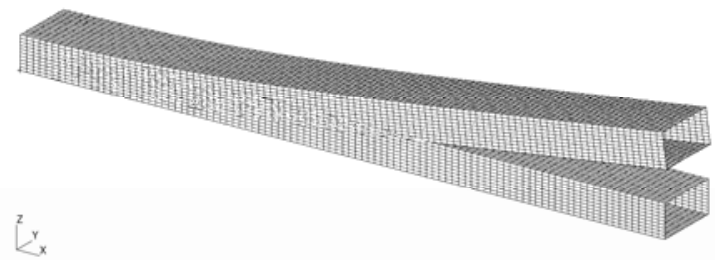

Shell Model. Mode \#1

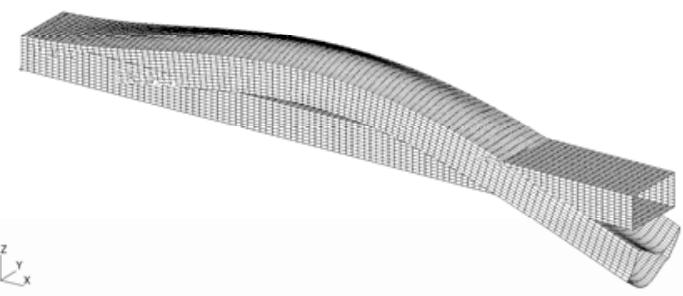

Shell Model. Mode \#3

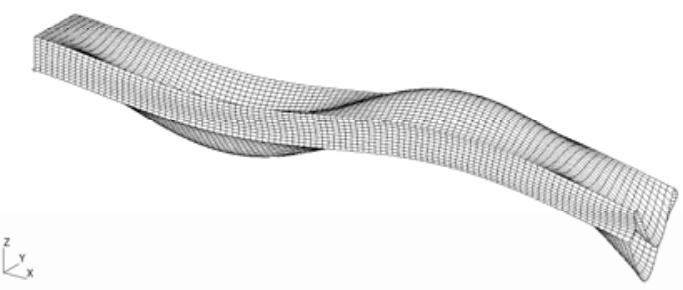

Shell Model. Mode \#9

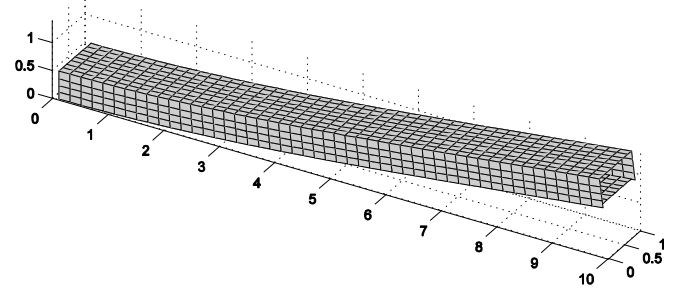

1-D with finite-section. Mode \#1

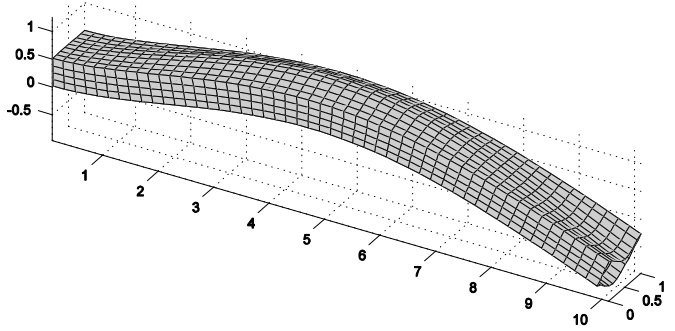

1-D with finite-section. Mode \#3

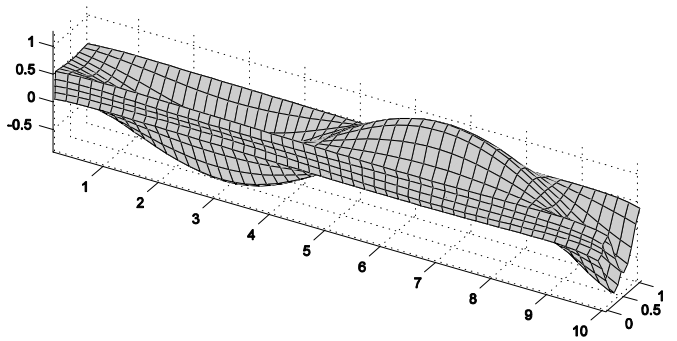

1-D with finite-section. Mode \#5

Figure 9. Vertical bending mode shapes for laminate $A$. 


\section{Lateral bending modes}

The natural frequencies of the first two lateral (chordwise) bending modes obtained by the different structural models for laminate $A$ are shown in Table 6 . The first lateral bending modes involve displacements of the relatively stiffer lateral walls and they show no significant local flexibility effects. Therefore we are in a situation in which Timoshenko's (BEST) description of the 1-D deformation provides a very good approximation to the free vibration characteristics.

Table 6. Natural frequencies (in $\mathrm{Hz}$ ) for laminate $A$. Lateral bending modes.

\begin{tabular}{ccccccccccc}
\hline \hline \multicolumn{2}{l}{ Shell } & \multicolumn{3}{c}{ 1-D Euler-Bernoullii } & \multicolumn{3}{c}{ 1-D Timoshenko } & \multicolumn{2}{c}{ 1-D with finite-section modes } \\
\hline$\#$ & Freq. & $\#$ & Freq. & MAC & $\#$ & Freq. & MAC & $\#$ & Freq. & MAC \\
\hline 2 & 5.61 & 2 & 5.63 & 0.994 & 2 & 5.59 & 0.994 & 2 & 5.62 & 0.994 \\
6 & 33.29 & 4 & 34.68 & 0.961 & 4 & 33.36 & 0.965 & 4 & 33.35 & 0.961 \\
\hline \hline
\end{tabular}

\section{Torsional modes}

The natural frequencies of the first two torsional modes for laminate $A$ are included in Table 7. Figure 10 shows the corresponding mode shapes. Table 7 also includes the correlation index (MAC number) between the different 1$\mathrm{D}$ formulations and the shell finite-element results.

Table 7. Natural frequencies (in Hz) for laminate $A$. Torsion modes.

\begin{tabular}{ccccccccccc}
\hline \hline \multicolumn{2}{c}{ Shell (2-D) } & \multicolumn{3}{c}{ 1-D Euler-Bernoulli } & \multicolumn{3}{c}{ 1-D Timoshenko } & \multicolumn{2}{c}{ 1-D with finite-section modes } \\
\hline$\#$ & Freq. & $\#$ & Freq. & MAC & $\#$ & Freq. & MAC & $\#$ & Freq. & MAC \\
\hline 4 & 25.21 & 5 & 48.67 & 0.386 & 5 & 48.67 & 0.386 & 4 & 31.31 & 0.714 \\
5 & 29.37 & 10 & 146.15 & 0.278 & 10 & 146.15 & 0.278 & 6 & 35.06 & 0.659
\end{tabular}

A substantial difference can be observed between the solutions given by the different 1-D formulations. This occurs because the description of the deformation used in standard beam theories (Euler-Bernoulli or Timoshenko) does not capture the relatively large warping deformations associated to the torsional dynamics of a thin-walled beam. A much better approximation was found by adding additional elastic degrees of freedom through finitesection modes, as it can be observed in Figure 10. In fact, due to the elastic uncoupling between torsion and bending modes for this layup, only mode \#241 in Figure 4 is actually needed to obtain the torsional vibration characteristics of the last column of Table 7.

In spite of the improvement on the estimation of the torsional modes when adding finite-section modes, there is still a $20 \%$ error in the estimation of the natural frequencies with respect to the shell model. There are two main reasons for this: 1) Finite-section modes are by definition approximations to the actual elastic field, and therefore carry some error with them. Adding additional elastic states may improve the results. 2) However, the main source for error in torsional modes is believed to be due to the fact that the present theory does not include Vlasov's warping constraint in the torsional curvature, as described for instance by Volovoi et al. ${ }^{16}$. This effect will be added in future expansions of the present theoretical framework. 


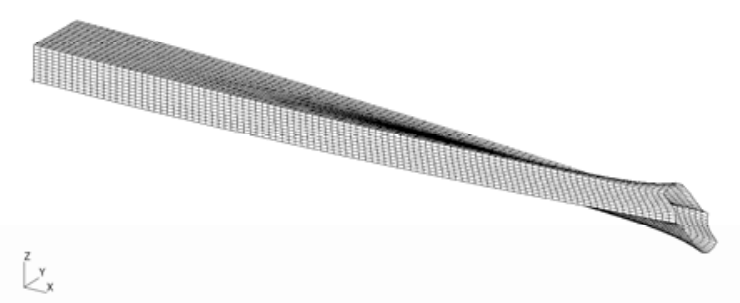

Shell Model. Mode \#4

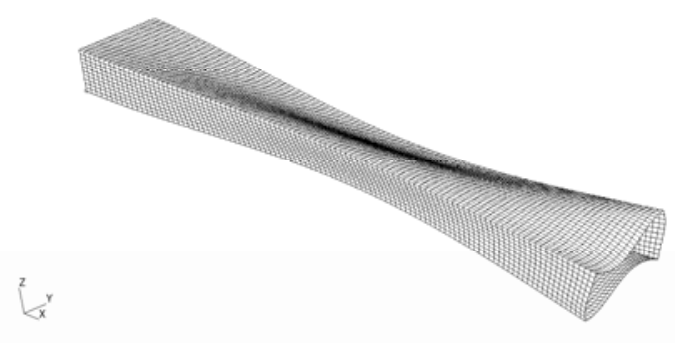

Shell Model. Mode \#5

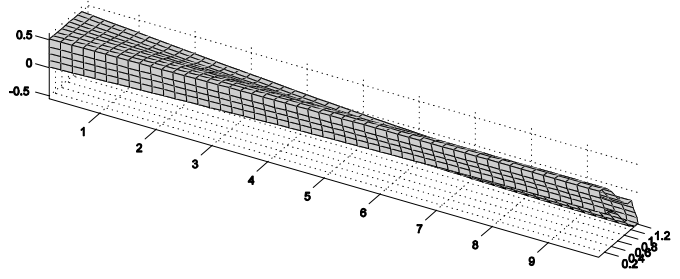

1-D with finite-section. Mode \#4

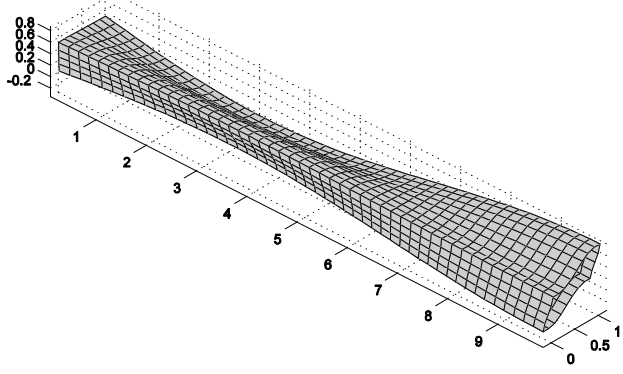

1-D with finite-section. Mode \#5

Figure 10. Torsion mode shapes for laminate $A$.

"Wall” modes

Finally, one can also identify a number of modes at low frequencies that essentially involve the motion of the walls. These modes are not captured by the standard Timoshenko beam description (BEST description) and will be refered here as "wall” modes. The natural frequencies of the first wall modes are shown in Table 8, as well as the comparison index between the corresponding eigenvectors obtained by the 1-D and the 2-D models. The actual mode shapes are included in Figure 11.

Table 8. Natural frequencies (in Hz) for laminate $A$. Wall modes.

\begin{tabular}{ccccc}
\hline \hline & Shell (2-D) & \multicolumn{3}{c}{ 1-D with finite-section modes } \\
\hline$\#$ & Freq. & $\#$ & Freq. & MAC \\
\hline 7 & 39.17 & 8 & 40.79 & 0.553 \\
8 & 39.92 & 9 & 41.37 & 0.494 \\
10 & 41.65 & 10 & 42.52 & 0.689 \\
11 & 42.95 & 11 & 44.21 & 0.845 \\
12 & 46.09 & 12 & 46.37 & 0.914 \\
\hline \hline
\end{tabular}

In general, an acceptable correlation can be observed among them, higher in the estimation of the vibration frequencies (with errors below 4\%) than on the modal shapes (with MAC numbers as low as 0.5). This has been done with only a few additional 1-D elastic degrees of freedom (i.e., the finite-section modes in Figure 5) that provide an extended characterization of the box-beam low-frequency dynamics using a 1-D structural model. As with the torsional modes, constrained warping at the clamped end is poorly captured by the 1-D model. The error decreases at higher frequencies, as they correspond to smaller wavelengths in the deformation, and subsequently to a propagation of the warping constraints to shorter distances along the beam. The effect of higher-order spatial derivatives of the finite-section amplitudes in reducing this error should be investigated in further extensions of this 
theory (this formulation was based on $q$ and $q^{\prime}$ contributions). As before, further refinement of these results is always possible by expanding the number of base Ritz functions in the approximation to the warping field.

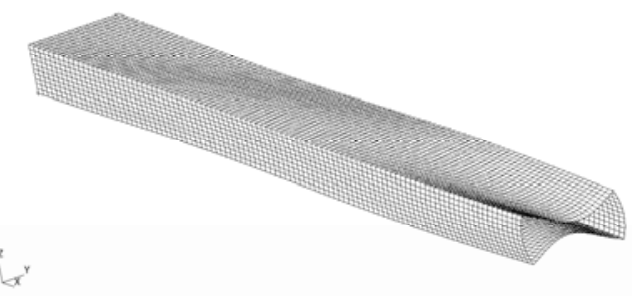

Shell Model. Mode \#7

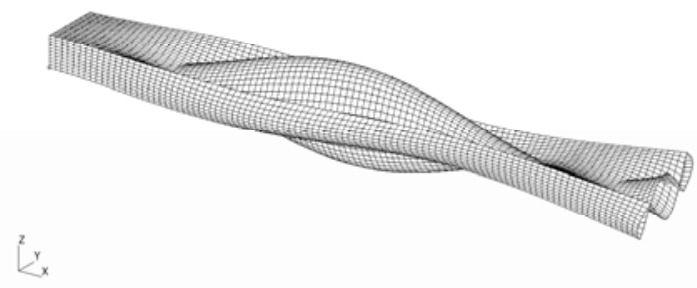

Shell Model. Mode \#8

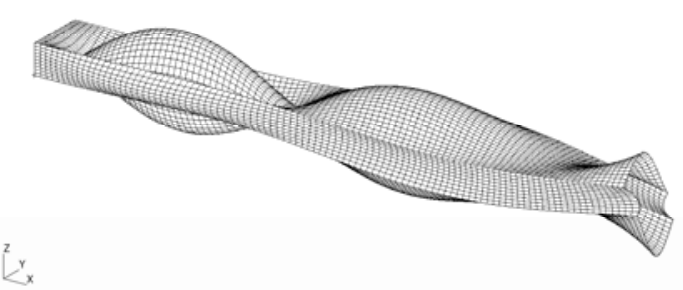

Shell Model. Mode \#10

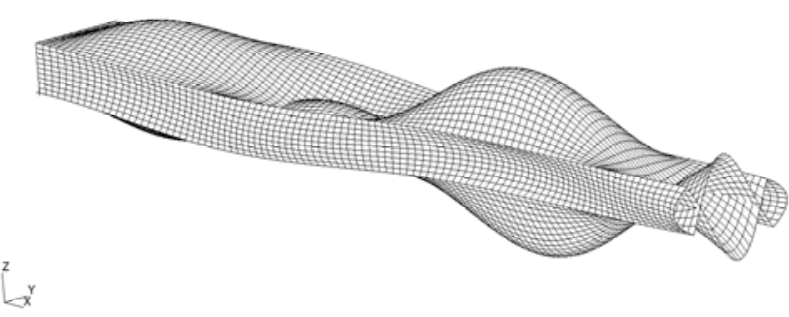

Shell Model. Mode \#11

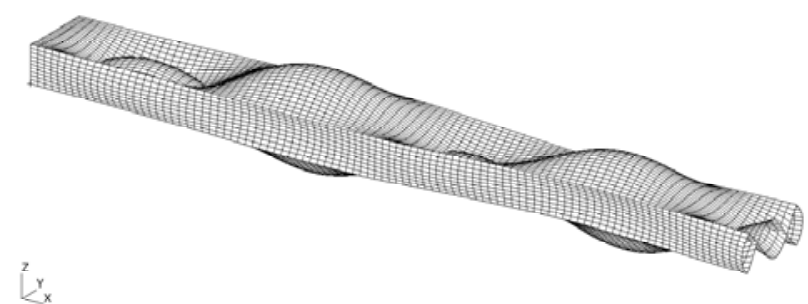

Shell Model. Mode \#12

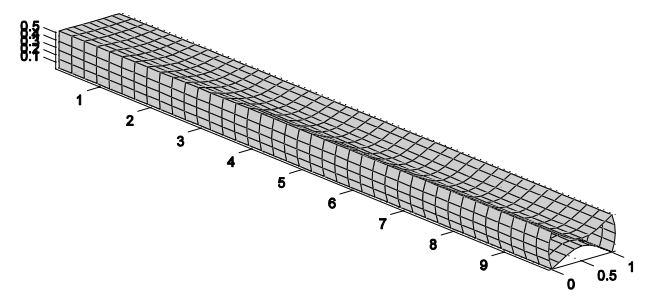

1-D with finite-section modes. Mode \#8

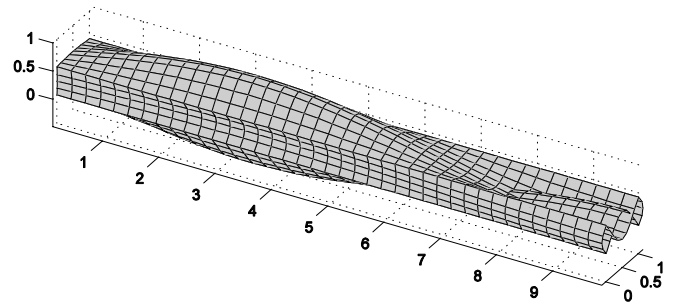

1-D with finite-section modes. Mode \#9

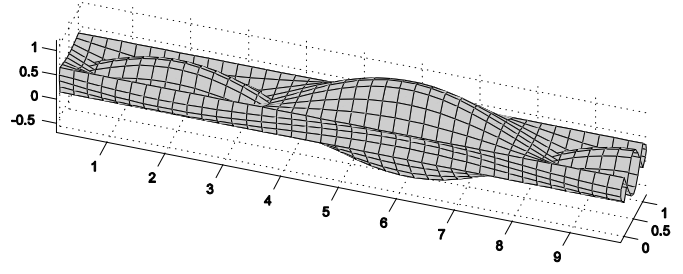

1-D with finite-section modes. Mode \#10

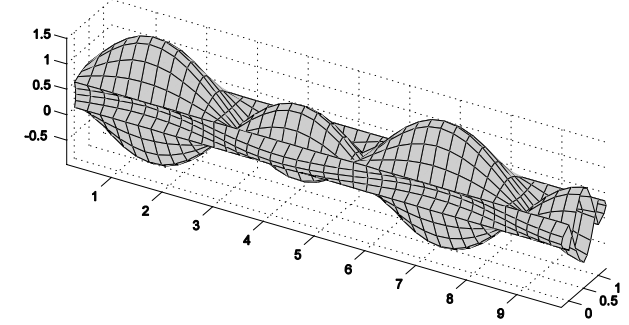

1-D with finite-section modes. Mode \#11

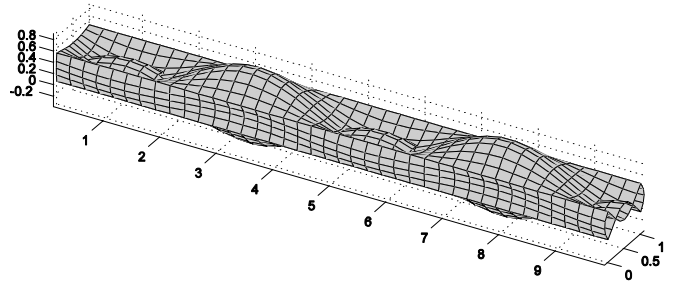

1-D with finite-section modes. Mode \#12

Figure 11. Wall mode shapes for laminate $A$. 
As a final remark, it should be noted that all previous low-frequency modes of a box beam can basically be obtained with only three of the finite-section modes presented in Figure 4 (modes \#122, \#124, and \#241). Furthermore, mode \#241 is linked to the twist curvature and they could be combined into a single elastic degree of freedom in the 1-D model. This however was not done here, to show the generality of the formulation.

\section{Concluding Remarks}

A beam model that allows arbitrary deformations of the finite-size cross sections has been introduced by expanding the conventional Timoshenko description with additional 1-D degrees of freedom. They have been named finite-section modes, and are a set of user-defined approximating functions to the warping displacement field. A spatial homogenization process has then determined the reduced 1-D description of the dynamics of a general anisotropic slender structure that, in addition to the conventional sectional forces and moments, it also includes the force conjugates to the amplitude of the finite-section modes. This resulted in a geometrically-nonlinear theory of beams with heterogeneous anisotropic material distribution on an arbitrary cross-sectional shape. Finite-element solutions at both the cross-sectional and longitudinal level define a flexible numerical framework for the analysis of complex configurations. For thin-walled composite beams, this refinement in the kinematic description may provide a significant improvement in accuracy with very little modeling or computational cost. Typical situations in which finite-section modes may be desirable are in the accurate computation of the deformation of composite beams under non-uniform distributed loads (for example, bimoments acting on a thin plate) and low-frequency dynamic response of composite beams at low frequencies. In those situations, a beam model would not need to be replaced by a shell model if a set of finite-section modes were defined. This is desirable in situations such as the aeroelastic modeling of composite rotorcraft blades, where 1-D models are commonly used.

Numerical results using this approach have been presented on an isotropic thin strip and composite box beam, and have compared very well with finite-element shell models. Only a few finite-section modes, which are defined numerically (in the examples, harmonic shapes for the box beam and parabolic deformation for the thin strip), are needed to obtain a rather complete description of the 3-D solid deformations, both under distributed loads and in the low-frequency linear vibration response. In the dynamic response, the new modes allow not only to capture vibration modes that are invisible by conventional beam models (wall modes), but also improved the results corresponding to the bending and torsion modes. This latter improvement can be explained because beam models based on the BEST (Bending-Extension-Shear-Twist) description are obtained by homogenization in the spatial variables of the 3-D solid mechanics equations, rather than in both space and time. This quasi-static approach to evaluate the internal energy neglects the inertia of the cross-sectional warping and is a source of error in the estimation of the vibration characteristics of the actual 3-D solid. Adding finite-section modes allows circumventing this limitation, as the contribution of warping deformation are included both to the strain and the kinetic energy of the beam. However, this is still done within the scope of a purely spatial cross-sectional reduction process, to ease both the theoretical formulation and the numerical implementation.

\section{Acknowledgments}

This work is supported by the Vertical Lift Rotorcraft Center of Excellence (VLRCOE) funded by the U.S. Army. Dr. Michael Rutkowski is the technical monitor.

\section{References}

\footnotetext{
${ }^{1}$ Volovoi VV, Hodges DH, Cesnik CES, Popescu B (2001). Assessment of Beam Modeling Methods for Rotor Blade Applications. Mathematical and Computer Modelling 33 (10-11), p 1099-1112.

${ }^{2}$ Jung SN, Nagaraj VT, Chopra I (1999). “Assessment of Composite Rotor Blade Modeling Techniques.” Journal of the American Helicopter Society 44 (3), p 188-205.

${ }^{3}$ Berdichevsky VL (1982). “On the Energy of an Elastic Rod.” PMM 45, p 518-529.

${ }^{4}$ Cesnik CES, Hodges DH (1997). "VABS: A New Concept for Composite Rotor Blade Cross-Sectional Modeling.” Journal of the American Helicopter Society 42 (1), p 27-38.

${ }^{5}$ Hodges DH (2005). Nonlinear Composite Beam Theory. Progress in Aeronautics and Astronautics, Vol. 213. AIAA, Reston, Virginia, USA.

${ }^{6}$ Buannic N, Cartraud P (2001). Higher-Order Effective Modeling of Periodic Heterogeneous Beams. I: Asymptotic expansion method. International Journal of Solids and Structures 38 (40-41), p 7139-7161.
} 
${ }^{7}$ Fan H, Widera GEO (1990). "Refined Engineering Beam Theory Based on the Asymptotic Expansion Approach.” AIAA Journal 29 (1), p 444-449.

${ }^{8}$ Parker DF (1979). “An Asymptotic Analysis of Large Deflections and Rotations of Elastic Rods.” International Journal of Solids and Structures 15 (5), p 361-377.

${ }^{9}$ Love AEH (1944). Mathematical Theory of Elasticity. Dover, New York.

${ }^{10}$ Gruttmann F, Wagner W (2001). "Shear correction factors in Timoshenko's beam theory for arbitrary shaped crosssections.” Computational Mechanics 27, p 199-207.

${ }^{11}$ Friedman Z, Kosmatka JB (2000). "Torsion and Flexure of a Prismatic Isotropic Beam Using the Boundary Element Method.” Computers and Structures 74 (4), p 479-494.

${ }^{12}$ Kosmatka JB (1992). "Extension-Bend-Twist Coupling Behavior of Non-Homogeneous Anisotropic Beams with Initial Twist.” AIAA Journal 30 (2), p 519-527.

${ }^{13}$ Giavotto V, Borri M, Mantegazza P, Giringhelli G, Carmaschi V, Maffioli GC, Mussi F (1983). “Anisotropic beam theory and applications.” Computers and Structures 16 (1-4), p 403-413.

${ }^{14}$ Palacios R, Cesnik CES (2005). "Cross-Sectional Analysis of Nonhomogeneous Anisotropic Active Slender Structures” AIAA Journal 43 (12), p 2624-2638.

${ }^{15}$ Palacios R, Cesnik CES (2007). “On The One-Dimensional Modeling of Camber Bending Deformations in Active Anisotropic Slender Structures.” Paper to appear.

${ }^{16}$ Volovoi VV, Hodges DH (2000). “Theory of Anisotropic Thin-Walled Beams.” ASME Journal of Applied Mechanics 67 (3), p 453-459.

${ }^{17}$ Reissner E (1973). “On One-Dimensional Large-Displacement Finite-Strain Beam Theory.” Studies in Applied Mathematics 52 (2), p 87-95.

${ }^{18}$ Simo JC (1985). “Finite strain beam formulation: The three-dimensional dynamic problem - Part I.” Computer Methods in Applied Mechanics and Engineering 49 (1), p 55-70.

${ }^{19}$ Danielson DA, Hodges DH (1987). "Nonlinear Beam Kinematics by Decomposition of the Rotation Tensor." ASME Journal of Applied Mechanics 54 (2), p 258-262.

${ }^{20}$ Danielson DA, Hodges DH (1988). “A Beam Theory for Large Global Rotation, Moderate Local Rotation, and Small Strain.” ASME Journal of Applied Mechanics 55 (1), p 179-184.

${ }^{21}$ Hodges DH (1990). “A Mixed Variational Formulation Based on Exact Intrinsic Equations for Dynamics of Moving Beams.” International journal of solids and structures 26 (11), p 1253-1273.

${ }^{22}$ Hodges DH, Shang X, Cesnik CES (1996). "Finite Element Solution of Nonlinear Intrinsic Equations for Curved Composite Beams.” Journal of the American Helicopter Society 41 (4), p 313-321.

${ }^{23}$ Cesnik CES, Brown EL (2002). "Modeling of High Aspect Ratio Active Flexible Wings for Roll Control.” AIAA Paper 2002-1719.

${ }^{24}$ Cesnik CES, Sutyrin VG, Hodges DH (1996). "Refined Theory of Composite Beams: The Role of Short-Wavelength Extrapolation.” International Journal of Solids and Structures 33 (10), p 1387-1408.

${ }^{25}$ Hodges DH (2004). “Geometrically Exact, Intrinsic Theory for Dynamics of Curved and Twisted Anisotropic Beams.” AIAA Journal 41 (6), p 1131-1137.

${ }^{26}$ Le K.C. (1999). Vibrations of shells and rods. Springer, Berlin, Germany.

${ }^{27}$ Zienkiewicz OG, Taylor RL (2000). The Finite-Element Method, $5^{\text {th }}$ ed. Butterworth-Heinemann, Oxford, UK.

${ }^{28}$ Lehoucq RB, Sorensen DC, Yang C (1998). ARPACK Users' Guide: Solution of Large-Scale Eigenvalue Problems with Implicitly Restarted Arnoldi Methods. SIAM, Philadelphia, USA.

${ }^{29}$ Chopra I (2002). "Review of State-of-Art of Smart Structures and Integrated Systems.” AIAA Journal 40 (11), p 2145 -2187. 